\title{
Primary and metastatic peritoneal surface malignancies
}

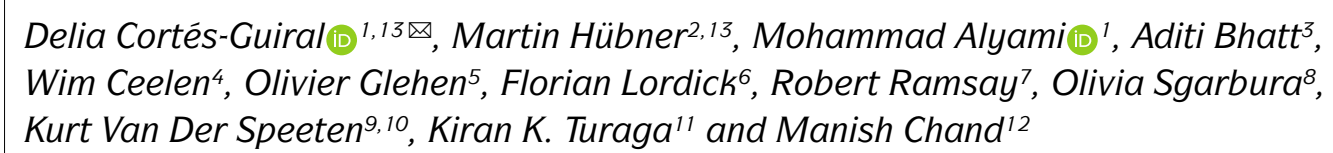

Abstract | Peritoneal surface malignancies comprise a heterogeneous group of primary tumours, including peritoneal mesothelioma, and peritoneal metastases of other tumours, including ovarian, gastric, colorectal, appendicular or pancreatic cancers. The pathophysiology of peritoneal malignancy is complex and not fully understood. The two main hypotheses are the transformation of mesothelial cells (peritoneal primary tumour) and shedding of cells from a primary tumour with implantation of cells in the peritoneal cavity (peritoneal metastasis). Diagnosis is challenging and often requires modern imaging and interventional techniques, including surgical exploration. In the past decade, new treatments and multimodal strategies helped to improve patient survival and quality of life and the premise that peritoneal malignancies are fatal diseases has been dismissed as management strategies, including complete cytoreductive surgery embedded in perioperative systemic chemotherapy, can provide cure in selected patients. Furthermore, intraperitoneal chemotherapy has become an important part of combination treatments. Improving locoregional treatment delivery to enhance penetration to tumour nodules and reduce systemic uptake is one of the most active research areas. The current main challenges involve not only offering the best treatment option and developing intraperitoneal therapies that are equivalent to current systemic therapies but also defining the optimal treatment sequence according to primary tumour, disease extent and patient preferences. New imaging modalities, less invasive surgery, nanomedicines and targeted therapies are the basis for a new era of intraperitoneal therapy and are beginning to show encouraging outcomes.
Peritoneal surface malignancies (PSM) comprise a heterogeneous group of quite different cancers in terms of incidence, sensitivity to systemic therapies and prognosis, all of which are unique in their proclivity for peritoneal dissemination. PSM can be primary tumours of the peritoneum (peritoneal mesothelioma and primary peritoneal cancer) or disseminate secondarily as peritoneal metastasis from tumours of other organs, which include those of intraperitoneal origin (tumours of the digestive and female reproductive tract as well as sarcoma) and those of extraperitoneal origin (lung, breast and kidney tumours) (FIG. 1).

Until 10 years ago, PSM were considered orphan diseases with limited therapeutic options and heralded a poor prognosis ${ }^{1}$. The primary reasons for poor patient outcomes are related to diagnosis at an advanced stage and the limited clinical response of most entities to conventional therapeutic options such as systemic chemotherapy. Major innovations over the past two decades include the adoption of novel surgical techniques, such as peritonectomy procedures and multivisceral resections to obtain complete cytoreduction (defined as absence of macroscopic disease) ${ }^{2}$, and the application of intraperitoneal chemotherapy to address microscopic residual disease ${ }^{3-5}$. Despite the perception of high morbidity of such procedures, optimization of perioperative care has led to the morbidity and mortality rates of these procedures being equivalent to those of other major abdominal cancer surgeries ${ }^{6-9}$. Concurrent development of new multidisciplinary strategies involving perioperative systemic chemotherapy ${ }^{10}$ and targeted and maintenance therapies ${ }^{11}$ has dramatically changed the landscapes and the prognoses of these diseases. In selected patients, long-term survival and even cure have become possible and the overall prognosis seems to be equivalent to that of patients with metastatic disease at other sites (such as in the liver or the lungs) ${ }^{12}$. In addition to therapies with curative intent, the development of less invasive and better-tolerated treatments can also provide symptomatic relief and improved survival for patients 


\author{
Author addresses \\ ${ }^{1}$ Surgical Oncology and General Surgery Department, King Khaled Hospital, Najran, \\ Saudi Arabia. \\ ${ }^{2}$ Department of Visceral Surgery, Lausanne University Hospital CHUV, University of \\ Lausanne (UNIL), Lausanne, Switzerland. \\ ${ }^{3}$ Department of Surgical Oncology, Zydus Hospital, Ahmedabad, India. \\ ${ }^{4}$ Department of Gl Surgery, Ghent University Hospital, Ghent, Belgium. \\ ${ }^{5}$ Surgical Department, Centre Hospitalo-Universitaire Lyon Sud, Pierre Bénite, France. \\ ${ }^{6}$ Department of Oncology, Gastroenterology, Hepatology, Pulmonology, and Infectious \\ Diseases, University Cancer Center Leipzig, Leipzig University Medical Center, Leipzig, \\ Germany. \\ ${ }^{7}$ Peter MacCallum Cancer Centre and the Sir Peter MacCallum Department of Oncology, \\ The University of Melbourne, Melbourne, Victoria, Australia. \\ ${ }^{8}$ Department of Surgical Oncology, Cancer Institute Montpellier, Montpellier, France. \\ ${ }^{9}$ Department of Surgical Oncology, Ziekenhuis Oost-Limburg, Genk, Belgium. \\ ${ }^{10}$ Faculty of Medicine and Life Sciences, BIOMED Research Institute, University Hasselt, \\ Hasselt, Belgium. \\ ${ }^{11}$ Department of Surgery, University of Chicago, Chicago, IL, USA. \\ ${ }^{12}$ Department of Surgery, University College of London, London, UK. \\ ${ }^{13}$ These authors contributed equally: Delia Cortés-Guiral, Martin Hübner.
}

with advanced disease and, therefore, a more optimistic outlook for patients and their families.

In this Primer, we describe the epidemiology, pathophysiology, diagnosis and prevention of primary peritoneal mesothelioma and primary peritoneal cancer, and of peritoneal metastases of the digestive tract, female reproductive tract and sarcoma as well as of extraperitoneal tumours. We discuss in detail the treatment options, with special emphasis on the quality of life (QoL) of patients with PSM, and close with an outlook on upcoming innovations.

\section{Epidemiology}

Incidence and prevalence of PSM vary widely based on the underlying primary tumour, with the highest incidence in patients with ovarian and gastric cancer ${ }^{13}$. As PSM are difficult to detect on cross-sectional imaging and not specifically documented in national registries as no separate code previously existed until recently, estimating their true incidence is difficult. Additionally, sites of metachronous metastases are not captured in most cancer registries, making it challenging to estimate the incidence of isolated peritoneal metastases. The GLOBOCAN registry, which provides estimates of the global incidence of cancer in 185 countries, does not record the incidence of PSM separately ${ }^{14}$. The closest approximation of these data comes from cohort studies that report the incidence or prevalence of peritoneal metastases as the proportion of patients with a particular histological subtyp $\mathrm{e}^{15,16}$. All data relating to the incidence and prevalence of PSM comes from high-income countries in the Western world. The incidence of most common cancers giving rise to PSM, such as ovarian, gastric and colorectal cancers, increases with age and they are more common in the age group $\geq 50$ years $^{14}$. In the past few decades, the incidence of these cancers has been increasing, especially that of colorectal cancer. However, the overall age-adjusted incidence of PSM seems to be mostly unchanged over the past four decades ${ }^{17}$. Of note, the incidence of secondary PSM by far exceeds the incidence of primary PSM. Between 2012 and 2016, the annual age-adjusted incidence of primary peritoneal malignancies was 4.36 per $1,000,000$ persons and that of synchronous secondary peritoneal metastases was 99.0 per 1,000,000 persons in the Czech Republic ${ }^{17}$.

\section{PSM secondary to intraperitoneal tumours}

The specific incidences of PSM vary depending on the underlying malignancy. Globally, the incidence of ovarian cancer and, therefore, of peritoneal metastases from ovarian cancer has increased in the past decade, with the highest average annual percentage change of 4.4\% found in Brazil, which may be associated with an increase in sedentary behaviour ${ }^{18,19}$. The reported relative incidence of peritoneal metastases from ovarian cancer is $60-70 \%$ whereas it is $<10 \%$ for other gynaecological malignancies ${ }^{20}$. Although studies on mortality specific to ovarian peritoneal metastases are lacking, overall mortality due to ovarian cancer has declined, most probably because more effective treatments have become available $e^{21,22}$

For gastrointestinal malignancies, the relative incidence of peritoneal metastases is highest for gastric cancer, at 15-43\% (for both synchronous and metachronous metastases), depending on gastric cancer subtype ${ }^{23,24}$. Although the incidence of gastric cancer is the highest in East Asia (Japan and Mongolia) and Eastern Europe, specific reporting of the incidence of peritoneal metastases from gastric cancer from these regions remains scarce $^{14}$. In a registry study from the Netherlands, peritoneal metastasis was detected in $14 \%$ of gastric cancers at the time of initial diagnosis, with a median survival time of 4 months $^{24,25}$. For colorectal cancer, the relative incidence of synchronous peritoneal metastases is $4-15 \%{ }^{16,26}$. Around $8 \%$ of patients at the time of primary resection and up to $25 \%$ of patients with recurrent colorectal cancer will develop metastatic disease confined to the peritoneal surfaces ${ }^{16,27}$. Appendiceal mucinous tumours are the most common underlying cause of pseudomyxoma peritonei (PMP), with a reported incidence of $0.4-1.9$ per 1,000,000 person-years ${ }^{28}$.

\section{PSM secondary to extraperitoneal tumours}

Compared with intraperitoneal tumours, extraperitoneal tumours rarely cause PSM, and data from population-based studies on the incidence of PSM from these cancers are very scarce. In a population-based study from Ireland, 5,791 patients were diagnosed with PSM from 1994 to 2021, of whom 543 (9\%) had an extra-abdominal primary malignancy ${ }^{29}$. Breast cancer (40.8\%), lung cancer (25.6\%) and melanoma (9.3\%) were the most common extra-abdominal cancers to develop PSM. The actual incidence of PSM from these cancers is low: $1.2 \%$ of 1,041 patients with lung cancer treated over a 26 -year period in a cohort study from Japan and $0.7 \%$ in 3,096 patients with breast cancer treated from 2001 to 2010 in a cohort study from the USA ${ }^{30,31}$.

\section{PSM of primary peritoneal tumours}

A population-based study from Sweden showed an increase of 0.9 to 1.24 per million per year in the incidence of peritoneal mesothelioma in the years 2011-2015 compared with 1993-2003 (REF. ${ }^{32}$ ). Occupational or environmental exposure to asbestos is a risk factor for 


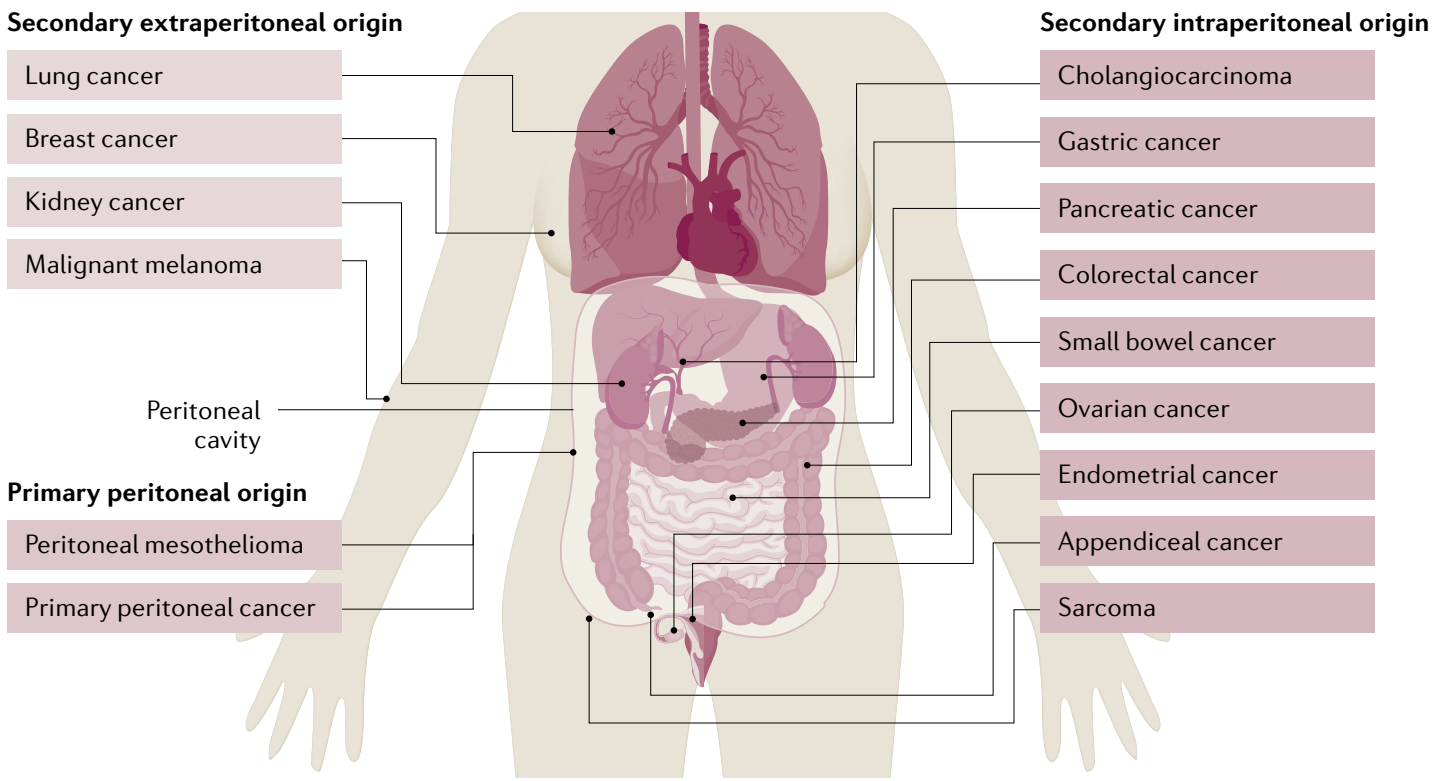

Fig. 1 | Tumours causing peritoneal surface malignancies. Peritoneal surface malignancies are a heterogeneous group of malignancies that can arise from primary tumours of the peritoneum or disseminate secondarily as peritoneal metastases from tumours of intraperitoneal origin and from tumours of extraperitoneal origin.

peritoneal mesothelioma and legislative measures banning its use since the 1980s (for example, in the USA, European Union and Russia) have resulted in a decline in the incidence of mesothelioma in these countries ${ }^{33,34}$. From 2008 to 2012, the world standardized incidence per 100,000 persons was 0.9 for men and 0.3 for women in the USA, and 1.7 for men and 0.4 for women in Europe. The incidence has declined in the USA since 2012 and in Europe since 2016. The decline is in the range of $10-20 \%$ and is seen more in men than in women. For other PSM, no single preventable risk factor can be associated with the risk of developing either the primary tumour or secondary peritoneal metastases.

\section{Trends in prognosis of PSM}

Randomized controlled trials and cohort studies show that the survival of patients with peritoneal metastases from various primary sites treated with a combination of locoregional and systemic treatment has improved compared with historical data from patients who received palliative treatment alone ${ }^{35-41}$. There are several reasons for this trend. The number of patients undergoing cytoreductive surgery (CRS) and hyperthermic intraperitoneal chemotherapy (HIPEC) has increased in the past three decades ${ }^{42-45}$. The increase in early diagnosis can be attributed to the improvement in imaging modalities and increased awareness ${ }^{46}$. Additionally, more effective systemic therapies for PSM have been developed ${ }^{47-49}$, enabling PSM resection in more patients. However, these data represent outcomes of subgroups of patients (mainly from high-income countries) and data from population-based studies are very limited.

A rare genetic condition resulting from a defect of $A P C$ leading to multiple polyps throughout the colon and associated with a near $100 \%$ risk of developing bowel cancer. involvement, histological subtype and positive peritoneal fluid cytology are some of the risk factors for peritoneal metastases that are common to most of the underlying primary tumours $\mathrm{s}^{50-52}$.

Over the past few decades, a high number of cancer risk genes for many gastrointestinal and gynaecological malignancies have been discovered. Around 10\% of colorectal cancers and $20-25 \%$ of ovarian cancers are associated with germline genetic disorders ${ }^{53,54}$. Defects in DNA repair pathways, such as homologous recombination repair and mismatch repair, are the most frequently described molecular mechanisms related to inherited cancers. Homologous recombination repair deficiency is often related to the $B R C A 1$ and/or BRCA2 mutations, whereas mismatch repair deficiency is commonly associated with Lynch syndrome. BRCA1 and BRCA2 germline mutations account for $15 \%$ of unselected epithelial ovarian cancers and are also associated with gastrointestinal cancers, such as pancreatic, colorectal and gastric cancer, but the clinical importance in gastrointestinal cancers is not yet clear. Lynch syndrome is characterized by a germline mutation in a mismatch repair gene (MLH1, MSH2, MSH6 or PMS2) or a germline deletion in EPCAM leading to inactivation of MSH2. Lynch syndrome accounts for $3 \%$ of colorectal cancers (usually located on the right side) but also for some extracolonic cancers such as endometrial, small bowel, gastric, hepatobiliary tract, ureteral and ovarian cancer ${ }^{60}$. Other mutations linked with colorectal cancer include APC involved in familial adenomatous polyposis ${ }^{53,55}$. Hereditary diffuse gastric cancer is linked to mutations of $C D H 1$, which encodes the cell-cell adhesion protein $\mathrm{E}$-cadherin ${ }^{56}$. Individuals with this genetic syndrome frequently develop signet ring cell carcinoma, which has a high risk of peritoneal metastases. For malignant mesothelioma, germline BAP1 mutations have been shown to increase the risk of developing peritoneal 


\section{Microvilli}

Small finger-like projections that increase the surface area of some cell types, including mesothelial cells.

Peritoneal cavity

The anatomical space in the abdomen and pelvis lined by peritoneum

\section{Mesothelial cells}

Specialized cells that line certain body cavities or organs these multifunctional cells have various functions, including the ubrication of surfaces and absorption of excess fluids mesothelioma after asbestos exposure ${ }^{57}$. Owing to the increased awareness of hereditary cancer risk, improved access to genetic counselling, surveillance and prophylactic risk-reducing surgery, a decrease in the incidence of these diseases and their secondary peritoneal metastases can be expected ${ }^{58-61}$.

\section{Mechanisms/Pathophysiology \\ Peritoneal anatomy and physiology}

The peritoneum is the largest and most complex serous membrane of the human body. The visceral peritoneum, covering the intra-abdominal organs and mesentery, forms a continuous layer with the parietal peritoneum, which lines the abdominal wall and pelvic cavities (FIG. 2). As a large sac, it covers abdominal organs that are tethered but still retain considerable mobility. The peritoneal surface area in adult women is $\sim 1.7 \mathrm{~m}^{2}$ (slightly more, on average, in men) but, when the enormous array of microvilli ( $\geq 300$ per mesothelial cell) is considered, the total area is likely much larger. This has important implications for the role of the peritoneum as a transport barrier in intraperitoneal chemotherapy ${ }^{62}$. The peritoneum is a closed-sac system in men, whereas it is an open-sac system in women where the fallopian tubes and ovaries are continuous with the peritoneal cavity. It is involved in the exchange of nutrients, metabolites and gases ${ }^{63}$, and serves as a barrier to infectious agents and as a line of defence through the transfer of innate and adaptive immune cells, cytokines and chemokines.

Most knowledge of the peritoneum is informed by studies in animal models ${ }^{64-69}$. Scanning electron microscopy and histological and immunohistochemical examination confirm that the morphology of the mammalian peritoneum is similar across species. Histologically, the peritoneum consists of a monolayer of mesothelial cells supported by a basement membrane that rests on a layer of connective tissue, also referred to as the submesothelium or stroma ${ }^{70}$. Morphologically, the mesothelial cells are predominantly squamous-like, flattened with microvilli but cuboidal mesothelial cells exist in some areas of the abdominal cavity. Mesothelial cells have both mesodermal (vimentin and desmin expression) and epithelial (cytokeratin expression) features ${ }^{71}$.

The peritoneum provides a slippery, non-adhesive surface through the microvilli of mesothelial cells, which produce large amounts of phosphatidylcholine and hyaluronic acid, forming the glycocalyx together with associated plasma protein ${ }^{72}$. Mesothelial cells produce humoral factors, such as complement C3 and C4 and human $\beta$-defensins, which, together with recruited cellular components of the immune system, comprise the peritoneal fluid protective mechanism ${ }^{73-76}$. The peritoneum also participates in immune responses against peritoneal pathogens that access the abdominal cavity. Mesothelial cells generate a chemotactic cytokine gradient from the basal to the apical side of the mesothelial lining, including IL-6 $\left(\right.$ REFS $\left.^{77-79}\right)$, IL-8 $\left(\right.$ REFS $\left.^{78,80}\right)$, IL-10 $\left(\right.$ REF. $\left.^{79}\right)$, IL-15 $\left(\mathrm{REF}^{81}\right)$, monocyte chemoattractant protein $1(\mathrm{MCP} 1)^{82}$ and stromal cell-derived factor 1 (SDF1) ${ }^{83}$. The mesothelial cell membrane also expresses receptors related to innate immunity such as Toll-like receptors ${ }^{80,82,84}$ and nucleotide-binding oligomerization domain (NOD)-like receptors $^{82}$. Leukocyte migration over the mesothelial lining is facilitated by integrins and adhesion molecules such as vascular cell adhesion molecule 1 (VCAM1) (V8,85-87 $^{78}$ and intercellular adhesion molecule 1 (ICAM1) ${ }^{78,87}$. Mesothelial cells participate in antigen presentation through presentation of major histocompatibility complex class II (MHC II) on their cell surface, both in an unstimulated state and after IFN $\gamma$ stimulation ${ }^{88-90}$.

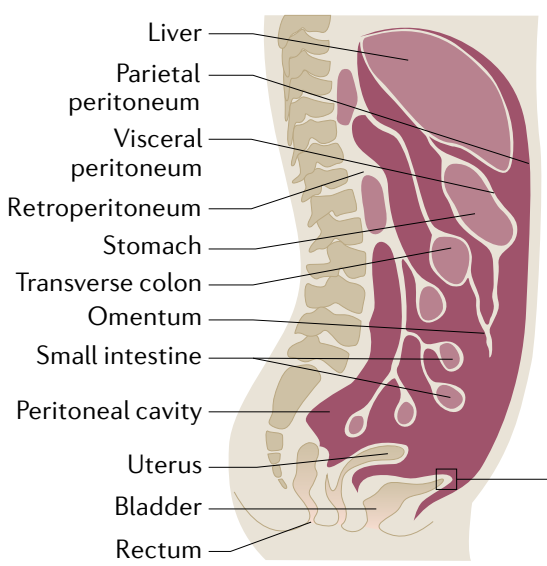

b

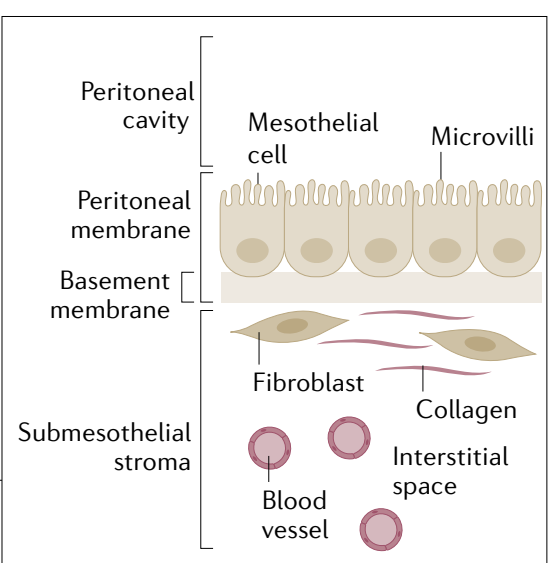

c

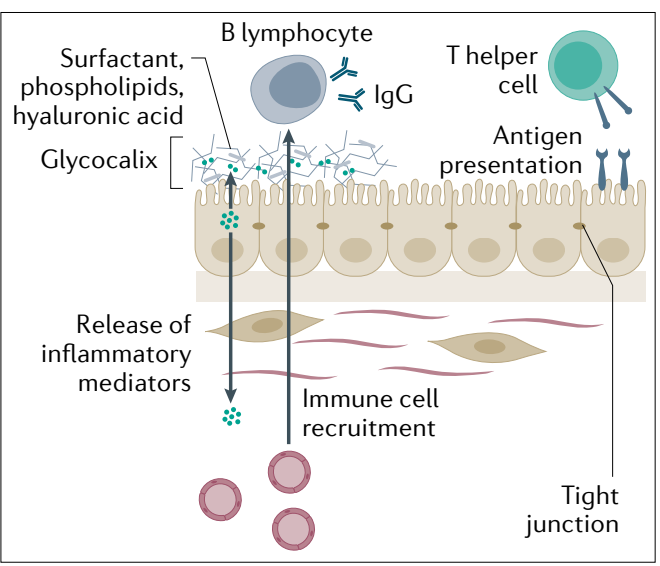

Fig. 2 | Peritoneal anatomy and physiology. a | The peritoneum is a serous membrane consisting of a single layer of mesothelial cells with complex apical and basal interactions. It forms a peritoneal sac that covers the abdominal organs. The visceral peritoneum describes the layer of the peritoneum adjacent to the abdominal organs, and the parietal peritoneum is the layer that adheres to the abdominal wall. The peritoneal cavity is the (virtual) space between these two layers that is filled with a small amount of serous fluid in the healthy state. $\mathbf{b} \mid$ The healthy peritoneal cavity is lined by mesothelial cells that express hundreds of microvilli per cell, which enable nutrient, waste and gas exchange as well as some organ mobility.
The mesothelial cells are supported by a basement membrane, which consists of a laminin polymer and a collagen IV network. Numerous other cells contribute to a dynamic submesothelial stroma that responds to mechanical stress, cellular damage and infection. c| Immune cells may traffic to the mesenchymal apical surface, which is protected by a complex chemical mix predominated by a glycocalyx, despite close cellular connections via tight junctions. Depending on the health status of the peritoneum and the presence of antigens, various inflammatory mediators can be released in both directions. Parts $\mathbf{a}$ and $\mathbf{b}$ adapted from REF. ${ }^{406}$, Springer Nature Limited. 


\section{Peritoneal injury and repair}

In the context of chronic (long-term peritoneal dialysis) or acute (surgical) peritoneal tissue injury, mesothelial cells have a dynamic role in tissue repair and scarring ${ }^{91,92}$ and regulate macrophage emigration from a site of inflammation ${ }^{90}$; they can promote procoagulant ${ }^{93,94}$, fibrinogenic and fibrinolytic mediators ${ }^{95}$. The repair process is facilitated by the production of an extracellular matrix (ECM) of type I, III, and IV collagen, elastin, fibronectin, laminin, and proteoglycans ${ }^{91,92}$. The repair process is modulated via expression of tumour necrosis factor $(\mathrm{TNF})^{96}, \mathrm{IL}-1 \beta^{97}$, epidermal growth factor $(\mathrm{EGF})^{98}$ and transforming growth factor- $\beta$ (TGF $\left.\beta\right)^{99,100}$. Overexpression of TGF $\beta$ in particular has been linked to the formation of adhesions and fibrosis ${ }^{101}$.

Clinically and experimentally, chronic inflammation of the peritoneal surface is observed following repeated and prolonged peritoneal dialysis for renal failure, which, in turn, gives rise to peritoneal fibrosis through the mechanism of epithelial-to-mesenchymal transition (EMT) of the mesothelial cells ${ }^{102}$. The TGF $\beta$ and hypoxia-inducible factor (HIF) pathways are involved in this process ${ }^{91,92}$ and are likely to be central to several diseases of the peritoneum. Specifically, EMT of mesothelial cells has also been suggested to be involved in the pathogenesis of peritoneal metastases ${ }^{103,104}$.

\section{Peritoneal metastasis and carcinomatosis}

Peritoneal metastasis and carcinomatosis development can be conceptualized as a stepwise process that starts with malignant cells gaining access to the peritoneal cavity. The origin of this malignant cell population can be situated either within the peritoneal cavity (most commonly from gastrointestinal cancer, ovarian cancer and malignant mesothelioma) or outside of the peritoneal cavity.

Detachment of cells from the primary tumour. In most patients, the first step in the cascade resulting in peritoneal metastases is shedding of tumour cells from the surface of the primary cancer (FIG. 3), which can occur spontaneously from locally advanced or perforated tumours, or can have iatrogenic causes ${ }^{105-108}$. Downregulation of cell-cell adhesion molecules, such as E-cadherin $(\mathrm{CDH} 1)$ via the transcription factor TWIST, promotes cancer cell detachment ${ }^{109,110}$. Loss of E-cadherin is a requisite for EMT, which results in a motile and invasive cellular phenotype ${ }^{111}$. Spontaneous shedding of loose cells is further facilitated by the elevated interstitial fluid pressure in most solid tumours ${ }^{112}$. This biomechanical property of malignant tissue is explained by rapid cellular proliferation, defective lymphatic drainage, fibrosis and contraction of the interstitial matrix, and increased osmotic pressure generated by anaerobic glycolysis and leakage of plasma proteins ${ }^{113,114}$. In addition, inadvertent cutting into tumour tissue or by sectioning blood, lymphatic or biliary channels that drain the tumour tissue has been shown to promote locoregional tumour cell dissemination ${ }^{115,116}$.

In some patients, peritoneal metastases arise from primary tumours outside of the peritoneal cavity such as lung cancer, breast cancer or malignant melanoma ${ }^{29}$. The pathophysiology of peritoneal spread from extra-abdominal primary cancers is not fully understood but must involve systemic (vascular and/or lymphatic) routes.

Peritoneal transport. Free cancer cells in the peritoneal cavity are subject to passive movement dictated by gravity and excursion of the diaphragm. As a result, cells usually follow a predictable path towards the pelvis and, from the pelvis, along the right paracolic gutter towards the sub-diaphragmatic spaces and the mesentery of the ileum ${ }^{117}$. Cancer cells also have active motility provided by lamellipodia and filipodia, whose mechanical force is generated by polymerization of actin microfilaments ${ }^{118}$. In ovarian and colorectal cancer, multi-cell clusters rather than isolated cancer cells can disseminate ${ }^{119,120}$. Presumably, these clusters may arise by aggregation of single cells or by shedding as clumps from the primary tumour.

Mesothelial adhesion. Free peritoneal cancer cells adhere to either the mesothelial lining or to the underlying ECM through specific adhesion molecules, including VCAM1, ICAM1 and PECAM1 (REFS ${ }^{78,121}$ ) (FIG. 3b,c). In vitro, mesothelial adhesion of colorectal tumour cells is mediated by the interaction of mesothelial ICAM1 and CD43 (sialophorin) rather than $\beta_{2}$ integrin, the most ubiquitous ligand of ICAM1 (REF. ${ }^{122}$ ). This interaction is exacerbated by the presence of damage to the mesothelial layer, whereby loss of cell junction integrity and mesothelial cells delaminate and expose the underlying basement membrane ${ }^{6,69}$.

In several cancer types, mesothelial adhesion was shown to be mediated by glycan-binding selectins expressed by mesothelial cells ${ }^{123-125}$. In addition, migrating cancer cells can be mechanically captured by neutrophil extracellular traps, a meshwork of decondensed DNA produced by activated neutrophils ${ }^{126}$. Adhesion between tumour cells and ECM components seems to be mediated primarily by the $\beta 1$ integrin subunit ${ }^{127}$. In the pathogenesis of ovarian peritoneal metastases, the ECM components versican and hyaluronan interact with CD44, the hyaluronan ligand expressed by ovarian cancer cells ${ }^{128}$. In addition, mesothelin, a glycoprotein physiologically expressed by mesothelial cells, was identified as a ligand for carbohydrate antigen 125 (CA125) and may have a role in peritoneal progression of ovarian cancer $^{129}$.

It is unclear why the omentum is a preferential site of peritoneal tumour growth ${ }^{130}$. The mechanisms underlying this tropism are not fully elucidated but it has been suggested that cancer growth is stimulated by the fatty acids stored in omental adipocytes and by the pro-angiogenic environment of the omental 'milky spots', which consist of immune aggregates and a dense capillary network ${ }^{131-133}$. Tumour cell binding may be mediated by a network of collagen I fibres overlaying the milky spots and by the expression of the pro-angiogenic vascular endothelial growth factor receptor 3 (VEGFR3) by omental microvessels ${ }^{134}$. In female patients, mucinous signet ring cell carcinomas at a location other than the 


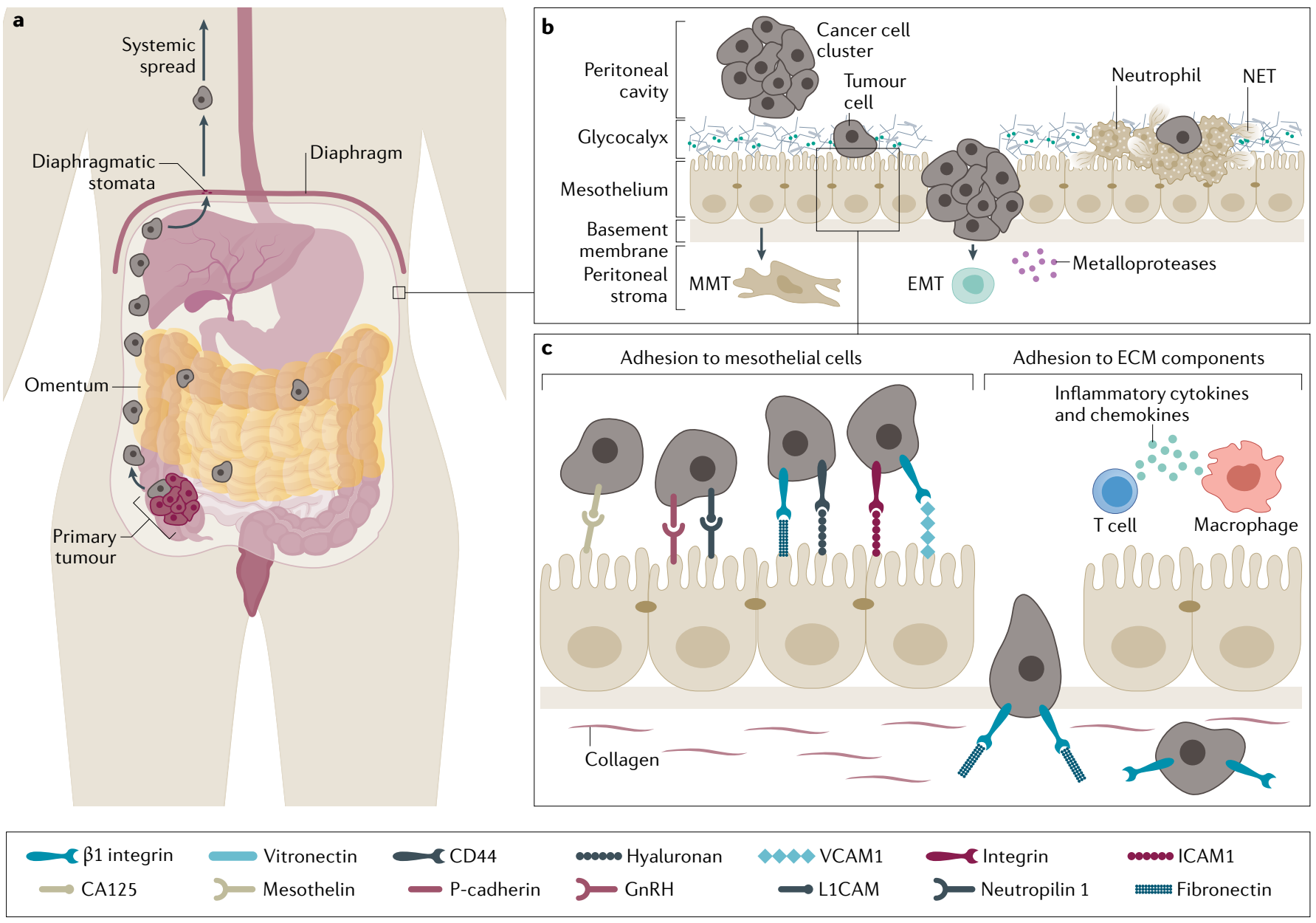

Fig. 3 | Metastatic spread to the peritoneum. a | The development of peritoneal carcinomatosis depends on mechanical forces, including gravity and diaphragmatic excursion, and on interactions between tumour cells, mesothelial cells and the underlying extracellular matrix (ECM). Individual or clusters of cancer cells are shed from the surface of the primary tumour (right colon cancer in this example). Subsequently, transcoelomic spread occurs, mainly in the direction of the pelvis and the right diaphragm. For reasons that are incompletely understood, there is a striking tropism for the greater omentum. At the surface of the diaphragm, the peritoneal cavity is in direct communication with lymphatic channels through stomata, enabling systemic dissemination of peritoneal metastases. $\mathbf{b} \mid$ Cancer cells present in the peritoneal cavity adhere to the mesothelial lining and to the underlying matrix through active (receptor mediated) and passive mechanisms. Tumour cells may become mechanically trapped in neutrophil extracellular traps
(NETs), expressed by activated neutrophils. In colorectal and ovarian cancer, collective invasion of clusters of cells has been reported. Invasion of the submesothelial stroma is facilitated by the action of metalloproteases and by mechanical or chemical damage to the mesothelial integrity. In addition, the process of epithelial-to-mesenchymal transition (EMT) provides cancer cells with a fibroblast-like, motile and invasive phenotype. Interestingly, some data suggest that the mesothelial cells also undergo mesenchymal transition (mesothelial-to-mesenchymal transition; MMT), enabling them to contribute to metastasis formation. c | Tumour cells express various receptors and ligands that enable close interactions with and binding to mesothelial cells and ECM components. The expression of these ligands and receptors is enhanced in the presence of inflammatory cytokines and chemokines. CA125, carbohydrate antigen 125; ICAM1, intercellular adhesion molecule 1; VCAM1, vascular cell adhesion molecule 1.
Pneumoperitoneum The consequence of air or $\mathrm{CO}_{2}$ filling the peritoneal cavity; the creation of artificial space is required for minimal invasive surgery. ovaries may give rise to ovarian metastases described as Krukenberg tumours. Depending on the primary malignancy, the pathogenesis of Krukenberg metastases may involve transcoelomic, lymphatic or haematogenous pathways $^{135}$

The expression of mesothelial adhesion molecules (and the resulting cancer cell adhesion) may be considerably enhanced by inflammatory stimuli induced by infection or surgical trauma ${ }^{136}$. For example, mesothelial expression of ICAM1 is stimulated by pro-inflammatory cytokines, including TNF, IL-1 $\beta$, IL- 6 and EGF ${ }^{137}$. Furthermore, malignant cells can become trapped in exudated fibrin matrices, and exudated plasma proteins, such as fibronectin and vitronectin, can act as bridging molecules between endothelial cells, smooth muscle cells and cancer cells via the $\alpha_{5} \beta_{1}$ integrin and $\alpha_{v} \beta_{3}$ integrin receptors $^{138}$.

Alterations in mesothelial binding may also be caused by mechanical factors. In vitro, elevation of the ambient pressure (for example, by establishing a pneumoperitoneum during surgery) increases adhesion of colon cancer cells to matrix proteins ${ }^{139}$. Additionally, elevated intraperitoneal pressure causes contraction of mesothelial cells, resulting in increased exposure of ECM binding sites ${ }^{69}$. Further to the mechanical effects on mesothelial structure, in preclinical studies, the acidification and dehydration associated with $\mathrm{CO}_{2}$ gas inflation during laparoscopic surgery promote tumour 


\section{Spiral CT}

High-resolution cross-sectional imaging modality on the basis of CT with a faster machine rotating continuously around the body, enabling more rapid acquisition and higher image resolution. growth and invasiveness but this has not been observed in the clinical setting ${ }^{140,141}$.

Invasion of the submesothelial tissue. Loose tumour cells gain access to submesothelial tissue at areas of peritoneal discontinuity or mesothelial cell contraction. Alternatively, tumour cells can induce apoptosis of mesothelial cells. For example, in vitro, colorectal cancer cells induced FAS-dependent apoptosis of cultured human mesothelial cells ${ }^{142}$. Once the mesothelial barrier is breached, tumour cells degrade the underlying matrix by the secretion of several proteases such as matrix metalloproteinases ${ }^{143,144}$. Interestingly, the phenotype and genotype of the established peritoneal metastases may differ substantially from those of the primary tumour. For example, gene expression studies of peritoneal tumours derived from colorectal cancer suggest preferential development of consensus molecular subtype 4 (CMS4) peritoneal tumours, representing cancers enriched for stromal and ECM elements ${ }^{145}$.

Systemic metastasis. Cancer cells that have disseminated to the peritoneal cavity can access the lymphatic system through specialized lymphatic stomata, which are localized mainly on the diaphragmatic surface, falciform ligament of the liver and pelvic side wall ${ }^{146}$. These stomata are $8-10 \mu \mathrm{m}^{2}$ round-to-oval gaps between cuboidal mesothelial cells and communicate directly with the lumen of a lymphatic vessel or lacuna ${ }^{147}$. In a rabbit model, passage of cancer cells from the peritoneal cavity via the stomata into the lymphatic cisterna was seen ${ }^{148}$. A similar observation was made in patients with gastric cancer, in whom passage of cancer cells into submesothelial lymphatic vessels was documented using scanning electron microscopy ${ }^{149}$. Importantly, both the density and diameter of the stomata and, therefore, the peritoneal absorptive capacity may increase by raised intraperitoneal pressure or by molecular mediators such as VEGF and nitric oxide ${ }^{150}$.

Symptoms associated with peritoneal metastases. The most common symptoms developing in patients with (extensive) peritoneal metastases include ascites formation, obstructive symptoms and pain. The pathophysiology of malignant ascites is complex and multifactorial and the result of an imbalance between peritoneal fluid production and absorption ${ }^{145}$. Obstruction of peritoneal lymphatics and stomata by invaded cancer cells impairs fluid resorption, while increased fluid filtration results from dilated peritoneal microvessels and enhanced vessel wall permeability, caused mainly by tumouroriginating $\mathrm{VEGF}^{151}$. Abdominal pain in patients with peritoneal metastases may be caused by ascites and the resulting abdominal distention, obstruction of the gastrointestinal or urinary tracts, and cancer infiltration of somatic and visceral afferent peritoneal nerves $^{145}$.

\section{Diagnosis, screening and prevention \\ Clinical presentation}

The clinical presentation of PSM varies depending on the origin and extent of the disease. At the onset, symptoms can be specific to the primary cancer for gastrointestinal malignancies, for which abdominal pain and distension are common in most patients. At late stage disease, at which ovarian cancer is diagnosed in $70 \%$ of cases, unspecific symptoms (abdominal distension, fatigue, nausea, anorexia, weight loss and constipation) increase in frequency in up to $85 \%$ of patients. Clinical examination may identify palpable mass and ascites as usual signs ${ }^{1,152}$.

\section{Imaging modalities}

Early diagnosis of PSM can be hampered by challenges in radiological detection. PSM imaging requires both modern technology and advanced reporting expertise ${ }^{153}$. Technological prerequisites include high spatial resolution for the often-small lesions combined with high contrast resolution (PSM have the same attenuation as normal peritoneum and bowel) and minimal motion artefact. An interobserver variability of $30-73 \%$ in CT detection sensitivity has been reported and appropriate specialty-specific training is lacking ${ }^{154}$.

In addition to its use in the diagnosis of PSM, imaging is a key factor in determining the surgical resectability of disease and predicting survival outcomes ${ }^{155,156}$. Anatomical sites that are crucial in assessing the feasibility of complete resection, such as small bowel mesentery and hepatic hilum, remain difficult to characterize ${ }^{153,157,158}$.

Ultrasonography has a limited role in diagnosis in general medical practice when concerning features, such as ascites or an omental cake, might trigger a high level of suspicion of underlying PSM. However, ultrasonography has no role in staging of PSM ${ }^{159}$, for which CT, PET-CT and MRI are preferred imaging modalities (TABLE 1).

Multidetector or spiral CT with multiplanar reconstruction has emerged as the primary imaging modality in PSM, facilitated by its widespread availability and high speed of acquisition. A meta-analysis reported a pooled sensitivity of $0.68(0.46-0.84)$ and a specificity of 0.88 (0.81-0.93) of CT in PSM ${ }^{160}$. The sensitivity of CT depends on the size and location of cancerous lesions. The detection rate of lesions $<0.5 \mathrm{~cm}$ is only $11 \%^{161}$ and

Table 1 | Imaging modalities and their performance for peritoneal surface malignancies

\begin{tabular}{|llllllr|} 
Imaging modality & Accuracy & $\begin{array}{l}\text { Positive } \\
\text { predictive value }\end{array}$ & $\begin{array}{l}\text { Negative } \\
\text { predictive value }\end{array}$ & Sensitivity & Specificity & Refs \\
\hline CT & 0.804 & 0.758 & 0.821 & 0.61 & 0.902 & $154-156,160-162$ \\
\hline PET-CT & 0.76 & 0.905 & 0.652 & 0.656 & 0.908 & $160,162,164,165$ \\
\hline MRI & 0.875 & 0.877 & 0.873 & 0.895 & 0.851 & $45,155,160,168$ \\
\hline
\end{tabular}

Although differences in appreciating peritoneal metastases of different malignancies are commonly described in the literature, these have never been quantified with accuracy or compared between different primary malignancies. 
CT accuracy is reduced owing to the complex anatomy in the pelvis, visceral peritoneum and right subphrenic space $^{162}$. For colorectal cancer PSM, the radiological peritoneal cancer index (PCI) by CT, as determined by a specialized radiologist in a PSM expert centre, correlates with the surgical or pathological PCI in only two-thirds of patients ${ }^{155}$. The PCI is the most accepted method of estimating tumour burden in the peritoneal cavity and is closely related to prognosis and success of CRS and HIPEC. The largest lesion in each of 13 anatomical sites in the peritoneal cavity is given a score of $1-3$ according to its size. This includes nine sites in the peritoneal cavity (sites 0-8) and four small bowel and mesenteric sites (sites 9-12). The sum of the scores gives a PCI of between 0 and 39 (REF. ${ }^{163}$ ).

PET-CT with the tracer ${ }^{18} \mathrm{~F}$-fluorodeoxyglucose $\left({ }^{18} \mathrm{~F}-\mathrm{FDG}\right)$ is an imaging modality that combines functional and morphological imaging techniques to increase accuracy. According to a meta-analysis, the sensitivity and specificity of PET-CT for PSM were $84 \%$ and $98 \%$, respectively ${ }^{164}$. It has a higher interobserver reproducibility than $\mathrm{CT}$ and helps in selecting potential candidates for CRS by excluding extra-abdominal disease. However, PET-CT has a longer acquisition time than CT and underperforms in mucinous PSM ${ }^{165}$.

ImmunoPET is a potentially paradigm-shifting molecular imaging modality combining the targeting capability of monoclonal antibodies and the inherent sensitivity of the PET technique ${ }^{166}$. Combining the same monoclonal antibody with a chemotherapeutic conjugate can leverage this imaging modality into a therapeutic strategy ${ }^{166,167}$ (FIG. 4). This strategy is currently under investigation for folate receptor- $\alpha$ (FRA)-based immunoPET and its therapeutic implications for epithelial ovarian cancer PSM $^{167}$.

The role of MRI has considerably evolved with the development of specific PSM imaging protocols ${ }^{45,153}$. Functional diffusion-weighted sequences have greatly added to morphological (T2-weighted and gadolinium-enhanced) sequences and improved PSM diagnosis, staging and follow-up ${ }^{168}$. The combination of CT and MRI improved the preoperative estimation of PCI and the diagnosis of non-resectability of PSM ${ }^{169}$. The use of MRI to identify small bowel involvement

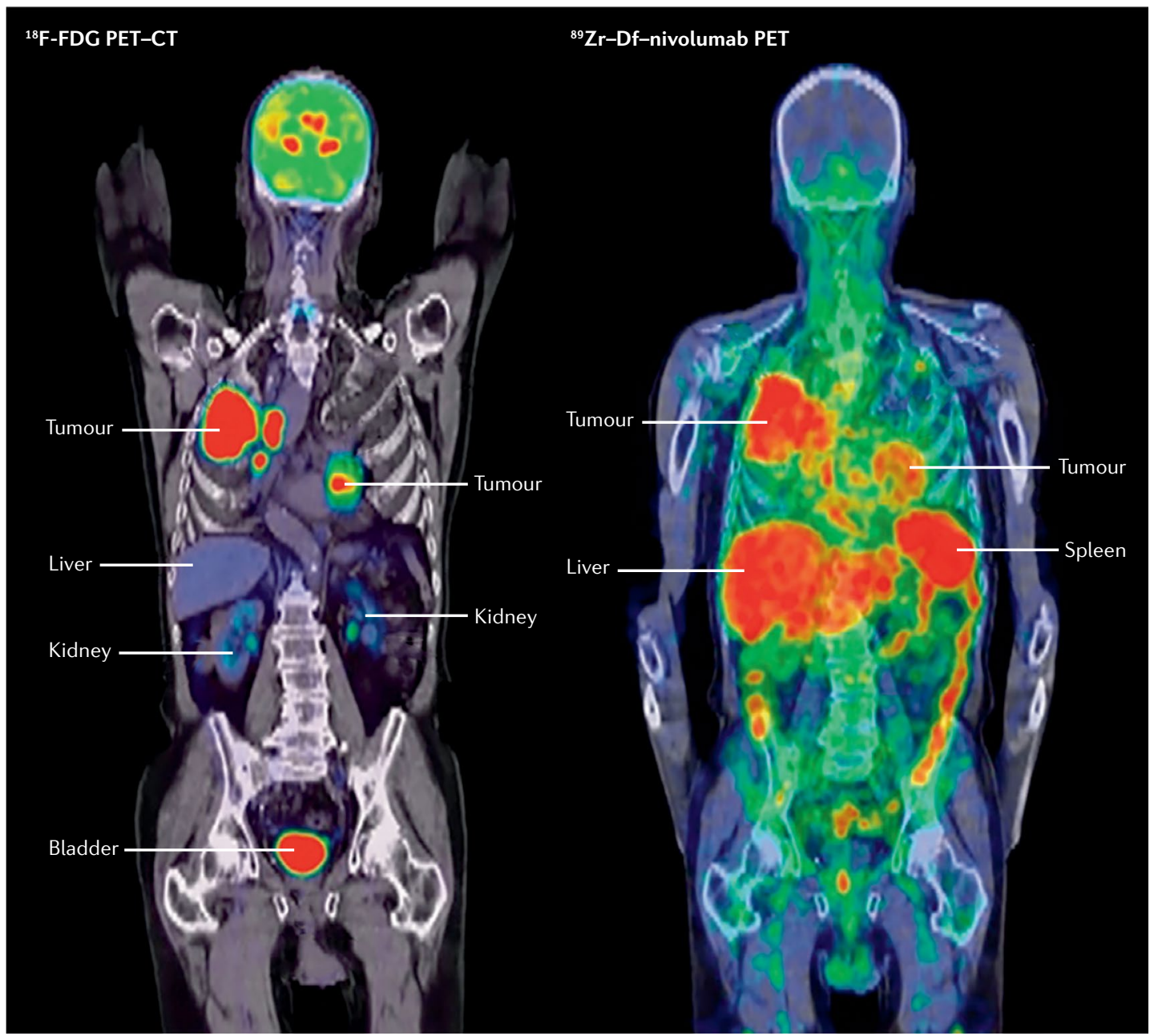

Fig. 4 | ImmunoPET imaging. Comparison of ${ }^{18} \mathrm{~F}$-fluorodeoxyglucose $\left({ }^{18} \mathrm{~F}\right.$-FDG) PET-CT (left image) with immunoPET (right image) for the same patient. Molecular imaging via immunoPET enables the combination of the precision of tissue targeting via a specific monoclonal antibody with the sensitivity of PET, resulting in better delineation of malignancy. 
benefits from a more experienced radiologist ${ }^{170}$ However, high costs, limited availability, motion artefacts, ascites and long learning curves restrict its widespread application. The emerging field of radiomics can further increase its role ${ }^{171}$.

\section{Tumour markers}

Tumour markers can be used in PSM diagnosis, prognosis and treatment response ${ }^{172}$. Routinely used tumour markers are carcinoembryonic antigen (CEA) and CA19-9 for gastrointestinal cancers at diagnosis and during follow-up. Diagnostic accuracies of CEA and CA19-9 are $66 \%$ and $71 \%$, respectively, in gastrointestinal tract malignancies ${ }^{173}$. CA125, which is highly specific for ovarian malignancies and mesothelioma can also be a useful marker of disease diagnosis and follow-up ${ }^{173-175}$. Serum CA125 and CA72-4 are clinically useful markers in diagnosis, evaluating the efficacy of chemotherapy and predicting the prognosis of patients with peritoneal dissemination from gastric cancer ${ }^{176}$.

In the context of PMP, when preoperative levels of CEA, CA19-9 and CA125 are not elevated, a complete CRS can be achieved in $97 \%$ of patients. Conversely, if these markers are elevated, the success of complete CRS drops to $50 \%{ }^{173,175,177}$. Finally, one important clinical use of tumour markers is the evaluation of chemotherapy efficacy, and some data suggest that the survival time of responders to chemotherapy (assessed by the four tumour markers CEA, CA19-9, CA125 and CA72-4) was longer than that of non-responders ${ }^{176,178}$.

\section{Endoscopy}

As PSM is often attributable to cancers of the upper or lower gastrointestinal tract, endoscopic procedures are a valuable diagnostic tool to determine the primary tumour location and obtain relevant biopsy samples ${ }^{179-181}$. In symptomatic patients, the thorough selection of patients for endoscopic examination increases the probability of obtaining relevant findings ${ }^{182,183}$. Endoscopy may enable the differentiation of extrinsic compression through disease from intrinsic stenosis in patients with PSM. That is, disease from outside the lumen of the gastrointestinal tract may lead to obstructive symptoms by pushing on the bowel compared with disease within the lumen of the bowel leading to a reduction in lumen size ${ }^{184}$.

\section{Surgical exploration}

Owing to the unspecific symptomatology and challenging radiological detection of PSM, surgical exploration can be beneficial in selected patients. Evaluation of the extent of the disease and assessment of its potential surgical resectability are the two major objectives of this approach ${ }^{185,186}$. Exploration is commonly undertaken in a minimally invasive manner using multi-port or single-port laparoscopy ${ }^{187}$. The extent of the disease throughout the peritoneal cavity is expressed through the PCI and can be established at different time points during disease management to identify occult PSM, decide the need for neoadjuvant therapy, or evaluate response to treatment and inclusion in clinical trials ${ }^{188}$. Diagnostic laparoscopy is required to establish the PCI and can exclude from surgery up to $54 \%$ of patients who have been classified as non-resectable ${ }^{189-194}$.

The rate of open-and-close laparotomies in which surgery is recognized to be futile owing to the presence of advanced disease is estimated to be $13-38 \%$ even after preoperative laparoscopic evaluation because of small bowel or porta hepatis involvement, which is difficult to assess by laparoscopy ${ }^{189-194}$. However, PCI evaluation by laparotomy remains the reference for patients with colorectal cancer PSM as laparoscopic evaluation failed to diagnose $18 \%$ of PSM in high-risk patients in one study ${ }^{195}$.

\section{Histological assessment and cytology}

Pathological sampling in PSM can be performed under radiological or laparoscopic guidance. Exploratory laparoscopy may be the more comprehensive technique for both cytology and histology as it enables multiple sampling ${ }^{196,197}$.

Pathological assessment is a key factor for the integrative management of peritoneal malignancies. At initial diagnosis, expertise in PSM is particularly required in rare peritoneal diseases such as PMP and peritoneal mesothelioma. Both entities have a high variability of pathological features resulting in borderline and malignant subtypes.

Histological assessment. In PMP, both the primary tumour usually located in the appendix ${ }^{198}$ and peritoneal dissemination are classified separately into up to four grades according to the Peritoneal Surface Oncology Group International (PSOGI) consensus for classification and pathological reporting of PMP and the WHO classification 2019 (REFS ${ }^{199,200}$ ).

For peritoneal mesothelioma, the histological classification distinguishes between diffuse malignant peritoneal mesothelioma and the borderline forms well-differentiated peritoneal mesothelioma and multicystic peritoneal mesothelioma ${ }^{201}$. The interobserver variation is small among expert pathologists but is not known for general pathologists ${ }^{202}$. Thus, PSOGI recommends a mandatory review of peritoneal mesothelioma specimens by a pathologist experienced in $\mathrm{PSM}^{201}$. Distinction among categories for both PMP and mesothelioma is crucial as it informs the choice of treatment.

For other primary cancers, the evaluation of PSM histological features includes sidedness and mutations. Sidedness of the tumour has prognostic relevance for both colorectal and gastric cancer, although its role is not clear for their $\mathrm{PSM}^{203-207}$. Out of the large panel of possible mutations, only few have therapeutic relevance, for example, microsatellite instability status in many gastrointestinal malignancies, which is associated with response to immunotherapy ${ }^{208}$. In metastatic colorectal cancer, microsatellite instability status, RAS mutations and BRAF mutations are routinely assessed ${ }^{209}$. Human epidermal growth factor receptor 2 (HER2) status was initially assessed in gastric cancer at any stage but is also becoming relevant in colorectal malignancies ${ }^{210,211}$. In ovarian cancer primary tumours, germline and somatic $B R C A 1$ and BRCA2 mutations have therapeutic relevance for poly(ADP-ribose) polymerase (PARP) 
FOLFOX

A systemic chemotherapy regimen including folinic acid (FOL), 5-fluorouracil (F), and oxaliplatin (OX), which can benefit from the addition of a targeted treatment such as an anti-epidermal growth factor receptor or an anti-vascular endothelial growth factor agent; different doses and duration of administration especially of 5-fluorouracil, are in use.

\section{FOLFIRI}

A systemic chemotherapy regimen including folinic acid (FOL), 5-fluorouracil (F) and irinotecan (IRI), which can benefit from the addition of a targeted treatment such as an anti-epidermal growth factor receptor or an anti-vascular endothelial growth factor agent. inhibition as is also the case with the homologous deficiency reparation assay that helps in selecting patients for this maintenance therapy ${ }^{212,213}$.

In The Cancer Genome Atlas, molecular subtypes were identified for some frequent malignancies, including primary colorectal and gastric cancer primary tumours $^{214,215}$. Some data indicate that the colorectal cancer subtype CMS4 is more frequently involved in PSM than other subtypes but no therapeutic implications have yet been established ${ }^{216}$. In advanced gastric cancer, molecular subtypes have a prognostic association with survival but their therapeutic relevance is currently limited ${ }^{217}$.

Treatment response can be assessed for different primary tumours and metastatic sites ${ }^{218}$. In PSM of gastrointestinal origin, the Peritoneal Regression Grading System (PRGS) scores the presence of residual tumour cells and regressive features and has demonstrated reproducibility ${ }^{219,220}$; however, the system has no correlation with survival and its value as a surrogate survival criterion is unknown. In PSM of gynaecological origin, including ovarian cancer, the chemotherapy response score is based on the presence of fibroinflammatory elements and/or the limited viability of the tumour cells and has shown prognostic value ${ }^{221,222}$.

Cytological assessment. Peritoneal cytology is a diagnostic and prognostic tool with low sensitivity owing to variability in sampling modalities ${ }^{223}$. Peritoneal lavage cytology is performed by introducing, stirring and aspirating from the abdominal cavity a variable quantity of saline solution but serous effusion cytology can also be performed in patients with ascites ${ }^{224}$. In gastric cancer PSM, it may guide treatment either as an indicator of response to neoadjuvant or intraperitoneal chemotherapy $^{38}$ or as a criterion for inclusion in prophylactic HIPEC and pressurized intraperitoneal aerosol chemotherapy (PIPAC) studies. In the combined progression index based on cytology and PRGS, positive cytology associated with a high PRGS is an independent factor of worse survival outcomes ${ }^{197}$. In most PSM, except those of gastric cancer origin, changes in treatment strategy on the basis of histological or cytological response are currently exploratory based on analogies with other metastatic sites.

\section{Prevention and screening}

In advanced colorectal cancer, there are high-risk (synchronous ovarian metastases and perforated primary tumour) and low-risk (T4 status, positive peritoneal lavage, mucinous subtype and signet cells) factors for developing metachronous PSM $^{225}$ as well as a predictive mode $^{226}$. Owing to the difficulty of establishing early diagnosis of PSM, several preventive strategies were designed and tested based on these factors.

The role of systemic chemotherapy in the prevention of metachronous colorectal cancer PSM is still unclear as very few trials of adjuvant systemic chemotherapy have investigated site-specific recurrence patterns. In an early study in patients at high risk with resected colorectal cancer receiving systemic FOLFOX-based or FOLFIRI-based adjuvant chemotherapy ${ }^{227}$, PSM were found at second-look surgery in the first year in $56 \%$ of 41 patients without any biochemical or radiological sign of recurrence. In a meta-analysis of data from 17,313 patients with pancreatic cancer receiving curative-intent pancreatic resection and systemic chemotherapy, $13.5 \%$ had initial tumour recurrence in the peritoneum ${ }^{228}$.

Another potential prophylactic strategy to prevent metachronous PSM is extensive intraoperative peritoneal lavage aiming to remove exfoliated tumour cells from the abdominal cavity. This approach has mostly been investigated in the context of high-risk gastric cancer for which positive lavage cytology is associated with an increased risk of developing metachronous peritoneal metastases ${ }^{229}$. In the EXPEL trial in 800 patients with gastric cancer randomly allocated to either gastrectomy alone or gastrectomy plus extensive intraoperative peritoneal lavage, the 3-year overall survival in the two groups was similar ${ }^{230}$.

In addition, intraperitoneal chemotherapy during surgery was hypothesized to be beneficial in preventing peritoneal metastases. In the early study in high-risk patients with resected colorectal cancer discussed above, 18 of 24 patients who were free of macroscopic peritoneal metastases at second-look received HIPEC and only 1 patient subsequently presented with peritoneal metastases ${ }^{227}$; by contrast, 3 of the 6 patients who did not receive HIPEC had peritoneal recurrence. Subsequently, the proactive, upfront approach of combining colorectal resection with HIPEC in high-risk colorectal cancer was investigated in two randomized controlled trials. However, prophylactic HIPEC with oxaliplatin failed to demonstrate superiority in reducing peritoneal metastases in the COLOPEC ${ }^{231}$ and PROPHYLOCHIP-PRODIGE 15 (REF. ${ }^{232}$ ) trials compared with standard follow-up after surgery ${ }^{233}$. Two more randomized trials, PROMENADE (oxaliplatin-based HIPEC) and HIPECT4 (mitomycin-based HIPEC), are ongoing ${ }^{234}$. For gastric cancer, recurrence at the peritoneal surface is common after curative-intent gastrectomy. Three randomized trials ${ }^{235-237}$ and several non-randomized trials investigated whether prophylactic HIPEC could reduce peritoneal recurrence and improve survival ${ }^{238}$. The studies demonstrated safety of this procedure and suggested decreased peritoneal recurrence and improved survival.

The identification of frequent mutations of cancer risk genes for many gastrointestinal and gynaecological malignancies has enabled the development of preventive strategies $^{239}$. Testing for mutations in BRCA1, BRCA2, $C D H 1$ and the DNA mismatch repair pathway has traditionally been guided by personal or family history ${ }^{240}$. Now, next-generation sequencing technology enables simultaneous assessment of many genes and the use of gene panels in clinical practice ${ }^{212}$. However, the use of this approach depends on economic availability and the socio-cultural perception of genetic heritage; for example, in some cultures, there may be reluctance to seek further information on genetic abnormality ${ }^{241}$.

National cancer screening programmes vary depending on the world region. Colorectal cancer screening is widely implemented and resulted in incidence and mortality reduction ${ }^{242}$. Gastric or oeso-gastric cancer 
Pseudomyxoma peritonei or resectable mesothelioma

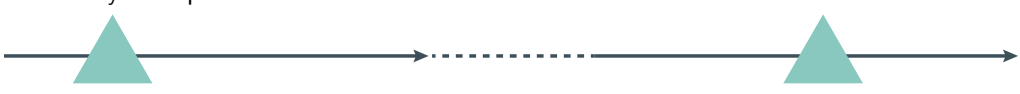

PSM of ovarian, colorectal or gastric cancers (predominant sequences)

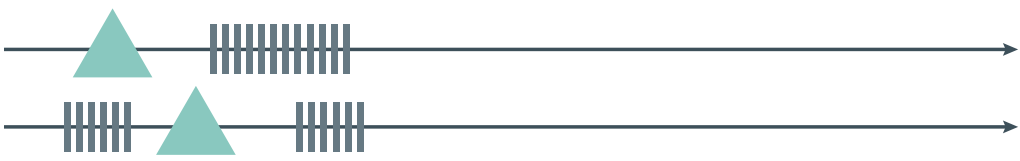

SPIC \pm systemic chemotherapy for PSM of ovarian or colorectal cancer

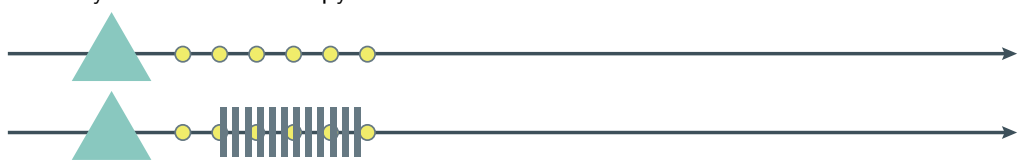

Palliative intent or borderline

PSM of ovarian, colorectal or gastric cancers (palliative)

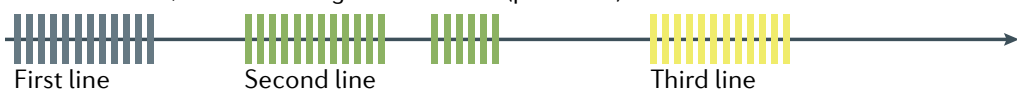

Predominantly peritoneal disease of ovarian, colorectal or gastric cancer origin

$\begin{array}{lll}\text { First line } & \text { Second line } & \text { Third line }\end{array}$

Non-resectable mesothelioma or isolated peritoneal disease of various origins

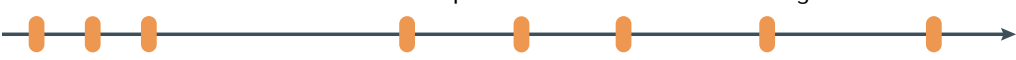

PSM of gastric or colorectal cancer (neoadjuvant bi-directional therapy)

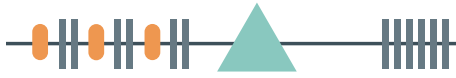

Non-resectable recurrence of mesothelioma

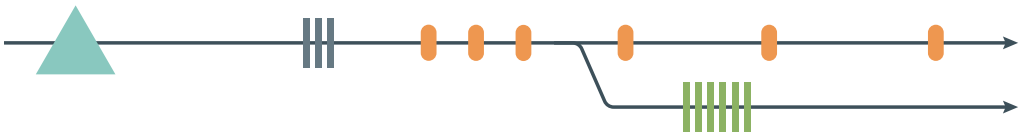

NIPS for advanced gastric and pancreatic peritoneal metastases

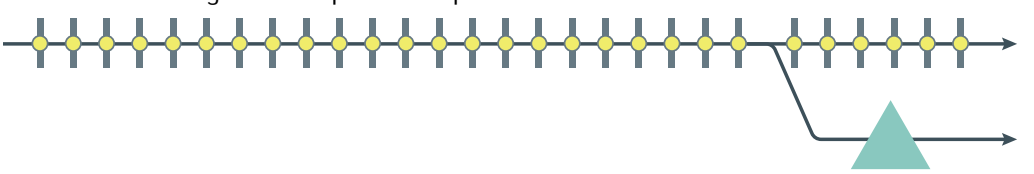

$\begin{aligned} & \text { Systemic } \\ & \text { chemotherapy }\end{aligned}$ CRS \pm HIPEC PIPAC $\quad 0 \begin{aligned} & \text { Catheter-based } \\ & \text { IP chemotherapy }\end{aligned}$

Fig. 5 | Typical treatment sequences for patients with PSM. The main treatment modalities currently used for the treatment of peritoneal surface malignancies (PSM) are systemic chemotherapy, cytoreductive surgery (CRS) with or without hyperthermic intraperitoneal chemotherapy (HIPEC), pressurized intraperitoneal aerosol chemotherapy (PIPAC) and catheter-based intraperitoneal (IP) chemotherapy. These treatments can be combined or applied sequentially according to the underlying primary tumour, the disease extent, and the individual patient's condition and preferences. These typical treatment sequences represent the most frequently encountered situations for patients with PSM. NIPS, neoadjuvant intraperitoneal and systemic chemotherapy; SPIC, sequential postoperative intraperitoneal chemotherapy.

screening is common in Asia and has led to reduced mortality from these cancers ${ }^{243}$. Earlier detection of these gastrointestinal cancers may have a beneficial effect on the incidence of PSM associated with these primary tumours.

Preventive strategies proposed to individuals with high-risk mutations include intensive screening and/or preventive surgery. For example, bilateral salpingo-oophorectomy can reduce the risk of epithelial ovarian cancer diagnosis by up to $96 \%^{244,245}$. The procedure should be performed earlier for those with BRCA1 mutations owing to the higher risk and earlier onset of the disease ${ }^{246}$. Prophylactic total colectomy is performed for those with familial adenomatous polyposis, usually before 25 years of age but is not recommended for Lynch syndrome ${ }^{60}$. Prophylactic total gastrectomy is recommended to the those with a $\mathrm{CDH} 1$ mutation at age 20-30 years or 5 years earlier than the age of the youngest affected family member ${ }^{247,248} ; 87 \%$ of patients who undergo prophylactic gastrectomy owing to $\mathrm{CDH} 1$ mutation have evidence of malignancy ${ }^{247,249}$.

\section{Management \\ Treatment modalities}

Management of PSM is an interdisciplinary challenge, often requiring individually adapted treatment concepts and optimized patient selection. The overall treatment strategy should evaluate curative management and should be discussed in a multidisciplinary tumour board to define adapted treatment sequences (FIG. 5). Typical modalities used in PSM management include systemic therapy, locoregional treatment (CRS and intraperitoneal chemotherapy), and supportive and palliative care measures.

Systemic therapy. Complications and sequelae of PSM, such as digestive disorders, cachexia and renal impairment, can challenge or prohibit effective systemic drug treatment ${ }^{250}$. An analysis of data from two prospective clinical trials of systemic chemotherapy in patients with colorectal cancer PSM showed reduced response rates of these metastases in comparison with those at other locations $s^{251}$. One potential explanation is the reduced blood supply to the peritoneum and, accordingly, to the diseased peritoneum ${ }^{252}$, which limits perfusion and drug delivery. Preclinical data have shown the importance of angiogenesis for tumour growth and dissemination to the peritoneum. These findings have laid the basis for the clinical evaluation of agents targeting VEGF signalling pathways in patients with ovarian cancer and other cancers spreading to the peritoneaum such as colorectal or breast cancer ${ }^{253}$.

Specific therapeutic regimens tailored for PSM are scarce. However, systemic chemotherapy is an important component of the oncological strategy and depends on primary tumour origin, the extent of peritoneal spread, the option of CRS, and the patient's performance status and organ function. In addition, biologically targeted drugs and immunotherapy have become available and are now in broad use for the treatment of metastatic disease in general. Unfortunately, trials of systemic targeted treatments and immunotherapy specifically for peritoneal metastases are lacking. Subgroup analyses from large trials with broad inclusion criteria are typically exploratory and underpowered. Thus, knowledge about 


\section{Box 1 Intraperitoneal treatment modalities}

Intraperitoneal treatment modalities include hyperthermic intraperitoneal chemotherapy (HIPEC), pressurized intraperitoneal aerosol chemotherapy (PIPAC), neoadjuvant intraperitoneal and systemic chemotherapy (NIPS) and early postoperative intraperitoneal chemotherapy (EPIC), which have different characteristics and indications.

\section{HIPEC}

- Applied as single administration after cytoreductive surgery (CRS) by use of a perfusion machine. Circulation of the heated chemotherapy solution can be performed using either an open (termed Coliseum) or a closed technique for a duration of 60-120 min and at a temperature of $40-43^{\circ} \mathrm{C}$.

- Indications: Curative.

- Potential other indications: Palliative, neoadjuvant and adjuvant.

\section{PIPAC}

- Applied repeatedly by laparoscopy using a two-trocar technique. PIPAC is not combined with CRS. Administration of chemotherapy is achieved via a high-pressure injector and a procedure-specific aerosolizer, creating a therapeutic aerosol with improved distribution and tissue entry.

- Indications: Palliative.

- Potential other indications: Neoadjuvant, adjuvant.

\section{NIPS}

- Long-course combination treatment of intraperitoneal and intravenous chemotherapy using implanted catheter access ports.

- Indications: Neoadjuvant.

EPIC

- Administered typically after CRS and HIPEC by use of intraoperatively placed intraperitoneal catheters to extend intraperitoneal drug exposure over 5 days postoperatively.

- Indications: Adjuvant.

Comparison of main features, advantages and disadvantages

\begin{tabular}{lllll} 
Feature & HIPEC & PIPAC & NIPS & EPIC \\
\hline Potency of drug & ++ & + & +++ & +++ \\
Intraperitoneal concentration & ++ & ++ & ++ & ++ \\
\hline Duration of tumour exposure & + & + & +++ & +++ \\
\hline Depth of drug infiltration & +++ & +++ & ++ & ++ \\
\hline Frequency and duration & ++ & ++ & +++ & +++ \\
Drug distribution & +++ & +++ & ++ & ++ \\
Combination with heat & +++ & + & $\#$ & $\#$ \\
Minimally invasive surgery & + & +++ & $\#$ & $\#$ \\
\hline Repeated pathological & + & +++ & $\#$ & $\#$ \\
evaluation of tumour response & & & & \\
Cost & +++ & ++ & + & + \\
\hline Potential toxicity & + & + & ++ & ++
\end{tabular}

+, low; ++, medium; +++, strong; \#, not applicable.

the efficacy of specific targeted drugs or immunotherapy are scarce.

Endocrine therapy, including in the form of selective oestrogen-receptor modulators, oestrogen-receptor blocking agents or aromatase inhibitors, combined with cyclin-dependent kinase 4 and 6 (CDK4 and CDK6) inhibitors, is an option in hormone-dependent cancers such as in breast cancer ${ }^{254}$. HER2-directed treatment is available for HER2 ${ }^{+}$breast and gastric cancers ${ }^{255,256}$. PARP inhibitors are used in the treatment of cancers that are deficient in their DNA damage response such as epithelial ovarian cancer or pancreatic ductal adenocarcinoma with BRCA1 and/or BRCA2 mutations ${ }^{257,258}$. Tropomyosin receptor kinase (TRK) inhibition is possible in cancers with TRK fusions ${ }^{259}$, and immune-checkpoint inhibition is used in colorectal and non-colorectal cancers with high microsatellite instability ${ }^{260,261}$.

Of note, molecularly targeted treatments and immunotherapy are dynamically evolving fields, and enabling access for patients to clinical research projects and drug therapy studies is important. Specialty-specific oncologists should be involved in multidisciplinary treatment considerations. The treatment centre should have access to a qualified molecular tumour board to discuss and recommend molecularly stratified and personalized treatment according to the ESMO Scale for Clinical Actionability of Molecular Targets (ESCAT) guidelines ${ }^{262,263}$. The ultimate goal is to ensure best outcomes for patients whose tumours display actionable molecular alterations.

Cytoreductive surgery. CRS is the principal component of curative treatment in PSM and aims to resect all visible tumour implants within the abdomen. Peritonectomy procedures and visceral resections are performed to surgically eradicate cancer on peritoneal surfaces ${ }^{264}$. The surgery comprises midline laparotomy and starts with an exhaustive exploration of the peritoneal cavity to evaluate the disease extent through the $\mathrm{PCI}^{187}$. At the end of surgery, completeness of cytoreduction according to the CC score (CC-0, no residual nodule; CC-1, $<2.5 \mathrm{~mm}$; CC-2, $<25 \mathrm{~mm}$; and CC-3, $>25 \mathrm{~mm}$ ) must be determined ${ }^{186}$. Postoperative surgical and medical complications are routinely evaluated within 90 days according to the Clavien-Dindo classification or the National Cancer Institute Common Terminology Criteria for Adverse Events (NCI-CTCAE) ${ }^{265,266}$. In selected patients, a laparoscopic and minimally invasive approach can be used ${ }^{267}$. Morbidity and mortality following CRS in large cohort studies differ between centres (15-50\% and $0.8-5 \%$, respectively $)^{268,269}$ but were estimated to be between $25-27 \%$ and $0-2 \%$, respectively, in prospective randomized controlled trials ${ }^{36,45}$. These rates are close to those reported for other types of major surgery ${ }^{6}$. Morbidity and mortality can be considerably decreased in high-volume centres and by optimizing perioperative care using standardized pathways $\mathrm{s}^{7,9}$.

Intraperitoneal chemotherapy. Several modalities of intraperitoneal chemotherapy can be used in patients with PSM (BOX 1) and can be combined into sequences (FIG. 5). HIPEC can be used in selected patients immediately at the end of CRS if complete resection was achieved or as a palliative treatment to control the $\operatorname{ascites}^{270}$. Early postoperative intraperitoneal chemotherapy is an option during the early postoperative period before adhesions develop. It has been in use for colorectal cancer with peritoneal metastases and for ovarian cancer with peritoneal metastases ${ }^{271}$. It can also be used in combination with CRS and HIPEC to treat patients with PSM of multiple origins, including gastric, colorectal and appendiceal cancers, as an additional therapy ${ }^{5,272}$. Intraperitoneal chemotherapy can also be 
delivered as a neoadjuvant treatment combined with systemic chemotherapy ${ }^{273}$ or as an adjuvant treatment via an intraperitoneal port ${ }^{274}$. PIPAC describes a modality in which agents are administered via aerosolization at the point of laparoscopy ${ }^{3}$.

Commonly reported adverse effects from the use of intraperitoneal chemotherapy agents are bleeding, nephrotoxicity, haematological toxicity and some rare presentation of allergic reaction in addition to the adverse events related to $\mathrm{CRS}^{36,275-278}$.

\section{Disease-specific management}

Management strategies differ according to the malignancy that caused PSM (Supplementary Tables 1, 2). The multidisciplinary tumour board usually select the treatment sequences of perioperative chemotherapy, surgery, and intraperitoneal chemotherapy and define the neoadjuvant and adjuvant therapy according to the type of PSM, while considering whether treatment has curative or palliative intent.

PMP and appendiceal cancer. CRS and HIPEC offer the best outcome for PMP and mucinous appendiceal tumours $^{279-281}$. As many patients with PMP present with extensive disease, adequate surgical skills and experience are required to balance the extent of surgery and the risk of complications. This complex treatment has a surgical learning curve with a peak reached after approximately 130 procedures ${ }^{282}$. Following complete CRS and HIPEC, prognosis is highly dependent on the histopathological characteristics of the tumour. In one analysis, median survival was not reached in those with low-grade PMP, whereas it was $<30$ months in those with high-grade $\mathrm{PMP}^{35}$. A large retrospective propensity analysis reported the beneficial effect of CRS combined with HIPEC compared with CRS alone ${ }^{283}$. This benefit was seen regardless of residual disease or histopathological grade. Various HIPEC protocols were used and survival advantages were reported with intraperitoneal cisplatin plus mitomycin C or intraperitoneal oxaliplatin plus intravenous 5-FU combinations.

Some patients present with histologically low grade but non-resectable, non-metastatic PMP and slow-growing abdominal tumours causing bowel obstruction that require total parenteral nutrition. Multivisceral transplantation may be an option for strictly selected patients and should involve teams specialized in PSM and in transplantation ${ }^{284}$.

Recurrence is common in PMP and the progressive accumulation of mucin with poor response to systemic treatments is debilitating. The combination of bromelain and acetylcysteine (BroMac) seems to have synergistic activity in the dissolution of tumour-produced mucin in the preclinical setting ${ }^{285,286}$. The first clinical study reported considerable mucolytic activity and a manageable safety profile, giving hope for patients with inoperable PMP or recurrence ${ }^{287}$. New approaches, such as iterative intraperitoneal chemotherapy, have been explored in patients with high-grade, unresectable appendiceal cancer, including goblet cell adenocarcinomas, and demonstrated promising results; however, further investigations are necessary ${ }^{288}$.
Malignant peritoneal mesothelioma. Systemic chemotherapy has not been shown to be effective in prolonging survival in malignant peritoneal mesothelioma $(\mathrm{MPM})^{289}$. The use of cisplatin or gemcitabine combined with the chemotherapeutic pemetrexed, which together constitute the standard therapy, resulted in a median overall survival of $\leq 27$ months ${ }^{290}$. The use of bevacizumab, a monoclonal antibody blocking angiogenesis by targeting vascular endothelial growth factor $\mathrm{A}$, can be considered following promising findings in pleural mesothelioma ${ }^{291}$. Immune-checkpoint inhibitors, such as nivolumab (anti-PDL1) and ipilimumab (anti-CLTA4), have demonstrated benefit in patients with pleural mesothelioma but their value in patients with MPM is incompletely studied ${ }^{292}$. Other targeted therapies, such as an anti-mesothelin antibody or pulsed dendritic cells, are promising but still under investigation ${ }^{293}$.

Combination treatment comprising CRS and HIPEC results in a median overall survival of 53 months in patients with MPM according to one multi-institutional analysis ${ }^{294}$. The main prognostic factors are tumour characteristics (histological subtype and Ki-67 expression), completeness of cytoreduction (CC score) and nodal status s $^{37295,296}$. CRS and HIPEC with cisplatin plus doxorubicin shows a trend towards a survival advantage and is recommended by PSOGI ${ }^{201}$.

For patients not amenable to complete CRS at initial diagnosis, the use of front-line intraperitoneal chemotherapy can be an option, such as PIPAC with cisplatin plus doxorubicin or intraperitoneal pemetrexed combined with systemic chemotherapy. Conversions to curative surgery have been reported in $>50 \%$ of patients ${ }^{297,298}$. One randomized trial, MESOTIP, is currently evaluating PIPAC as neoadjuvant treatment ${ }^{299}$. Long-term normothermic intraperitoneal chemotherapy using pemetrexed may also provide increased survival of $75 \%$ at 5 years $^{274}$.

PSM of colorectal origin. In colorectal PSM, data from an early randomized trial found a significant survival benefit with CRS plus mitomycin C-based HIPEC followed by systemic chemotherapy versus systemic chemotherapy alone (22.4 versus 12.6 months; $P=0.032$ ); other prospective cohorts have validated these results ${ }^{41,300-302}$. In addition, a significant survival benefit was seen in patients who had undergone CRS followed by intraperitoneal chemotherapy compared with those who received systemic chemotherapy alone (25 versus 18 months; $P=0.04)^{303}$. The PRODIGE 7 trial tested CRS plus oxaliplatin-based HIPEC compared with CRS alone and failed to demonstrate an improvement in overall survival or recurrence-free survival ${ }^{36}$. CRS combined with modern systemic chemotherapy in expert centres achieved a better than expected median overall survival of 41 months in the PRODIGE 7 trial $^{36}$. This finding highlighted the major role of completeness of CRS as the principal prognostic factor of patient outcome. HIPEC with high-dose oxaliplatin, which increases the risk of intraperitoneal bleeding ${ }^{275}$, and for a too short duration of $30 \mathrm{~min}\left(\mathrm{REF}^{233}\right)$ was not appropriate and should be abandoned following three negative phase III trials $^{36,231,304}$. Future trials should further investigate 
the role and optimal type of HIPEC in colorectal PSM management, which remains controversial ${ }^{305}$.

PSM of gastric origin. Peritoneal metastases are common in the late stage of gastric cancer ${ }^{24}$ and these patients have a poor prognosis despite systemic chemotherapy ${ }^{306}$. An analysis of results in prospective databases suggested a survival benefit of adding HIPEC to CRS in patients strictly selected for localized $\mathrm{PSM}^{307}$. Other studies also reported long-term survival following the use of HIPEC in patients with a CC-0 CRS and a PCI $<6\left(\right.$ REFS $\left.^{307-309}\right)$. New approaches for patients with gastric cancer and PSM, such as repeated HIPEC in a phase II trial, have demonstrated promising results and further trials are in progress ${ }^{310,311}$. The role of CRS and HIPEC compared with palliative chemotherapy is under evaluation in the PERISCOPE II phase III trial ${ }^{312}$. For non-resectable peritoneal metastases from gastric cancer, palliative intraperitoneal chemotherapy provided encouraging survival results. The combination of systemic chemotherapy with PIPAC using cisplatin and doxorubicin resulted in a median survival of 19.1 months and $14.3 \%$ of patients became eligible for curative procedures ${ }^{298,313}$. Neoadjuvant intraperitoneal and systemic chemotherapy or palliative intraperitoneal chemotherapy using docetaxel or paclitaxel seem to further reduce peritoneal progression and improve survival ${ }^{38,314-316}$. The PHOENIX-GC trial suggested a clinical benefit of intraperitoneal paclitaxel treatment ${ }^{38}$.

PSM of ovarian, fallopian tube and primary peritoneal cancer origin. Primary CRS followed by systemic chemotherapy is the standard of care for patients with PSM of ovarian, fallopian tube and primary peritoneal cancer origin. The term debulking surgery refers to a procedure in which the goal of optimal debulking is to leave residual disease $<1 \mathrm{~cm}\left(\mathrm{REF}^{317}\right)$. When complete CRS is not possible owing to disease extent or location, poor general health status or condition, neoadjuvant systemic chemotherapy should be delivered for 3-4 cycles before reconsidering indication for complete surgical resection (interval surgery $)^{318,319}$. Of note, the goal of CRS initially and at the interval setting should be complete removal of macroscopic disease ${ }^{320}$. The role of pelvic and para-aortic lymphadenectomy remains controversial. One randomized trial demonstrated that it can be safely omitted in patients without evidence of node involvement ${ }^{321}$.

Despite encouraging evidence for the use of HIPEC in combination with CRS from a meta-analysis of nine comparative studies ${ }^{322}$, the use of HIPEC is recommended only as an option at the interval setting in most countries. In this setting, the open-label phase III OVHIPEC trial demonstrated that HIPEC with cisplatin increased disease-free survival by $\sim 4$ months and overall survival by $\sim 12$ months without increasing morbidity ${ }^{45}$.

The benefit of CRS on overall survival in case of disease recurrence has been demonstrated in strictly selected patients with PSM of ovarian, fallopian tube and primary peritoneal cancer origin ${ }^{323,324}$. The criteria for selection of the best candidates for CRS include good performance status, platinum treatment-free interval of $<6$ months, complete resection at the primary surgery and absence of large ascites volumes. However, these criteria are only positive predictors if complete resection is achieved, which can be an option in specialized centres $^{325,326}$.

The role of CRS combined with HIPEC seems promising, especially for platinum-resistant ovarian cancer ${ }^{327,328}$. In one randomized study, CRS plus HIPEC followed by systemic chemotherapy versus CRS only followed by systemic chemotherapy resulted in a median survival of 19.4 months versus 11.2 months $(P<0.05)$, respectively ${ }^{327}$. Its role is being investigated also by the ongoing randomized controlled trial HIPOVA-01 (REF. ${ }^{329}$ ).

The utility of PIPAC with cisplatin and doxorubicin for recurrent ovarian, fallopian tube and peritoneal cancer PSM has been validated in a phase I study ${ }^{330}$. This treatment demonstrated safety and potential benefit as a palliative option in patients with recurrent disease: $62 \%$ of patients had an objective tumour response; histological tumour regression and PCI improvement were observed in $76 \%$ who underwent three courses of PIPAC; and no grade 4 adverse events or death related to treatment were observed ${ }^{331,332}$. This modality is being investigated in the ongoing phase III trial PIPAC-OV3 (REF. ${ }^{331}$ ).

PSM of rare origins. Limited data are available for cancers that rarely present with peritoneal metastases or that are rarely eligible for curative resection (mainly due to extraperitoneal dissemination) such as pancreatic, biliary tract, breast, lung and neuroendocrine tumours as well as sarcoma ${ }^{333}$. For these rare PSM, a worldwide analysis led by PSOGI observed promising, but sporadic, long-term survival in strictly selected patients in centres specialized in PSM management. Common selection criteria for curative procedures include the possibility of complete CRS, low PCI, no extra-abdominal metastases, favourable tumour biology or long-term control with systemic chemotherapy ${ }^{334}$.

For pancreatic peritoneal metastases, two case reports on treatment with CRS and HIPEC using mitomycin C showed overall survival of 48 and 70 months $^{335}$, but a small case series of seven patients treated with CRS and cisplatin-based HIPEC observed overall survival of 16 months, which was associated with a high rate of complications and did not alter disease progression ${ }^{336}$. Furthermore, in the PSOGI analysis, PSM of pancreatic origin was a negative prognostic factor ${ }^{333}$. For peritoneal metastases from cholangiocarcinoma, an analysis of a prospective multicentre database for 34 patients treated by CRS and HIPEC and 21 patients treated with systemic chemotherapy found a median overall survival of 21.4 and 9.3 months for the CRS and HIPEC group and the chemotherapy group, respectively ${ }^{334}$. Peritoneal metastases from breast cancer are extremely rare and $\sim 82 \%$ of patients with peritoneal metastases also have other metastatic sites ${ }^{31}$. A case series of 5 patients treated with CRS and HIPEC with a median elapsed time between breast cancer diagnosis and peritoneal disease of 18 years observed a 56-month overall survival ${ }^{337}$. Furthermore, a cohort study that included 73 patients with gastrointestinal metastasis, of whom 32 presented with PSM only, found that surgical resection did not 
considerably extend overall survival ${ }^{338}$. In the few reports for peritoneal sarcomatosis from different histotypes (7-60 patients $)^{339-343}$, overall survival was $12-34$ months and prognostic factors included completeness of CRS and the extent of peritoneal involvement according to $\mathrm{PCI}^{344}$. The PSOGI analysis included 189 patients with different histotypes, of whom $29 \%$ had 5 -year overall survival and $14 \%$ had 5 -year disease-free survival, concluding that the most prognostic factor was CRS and that the role of HIPEC remains to be determined ${ }^{333}$. Finally, neuroendocrine PSM mostly associated with extraperitoneal involvement negatively influences prognosis ${ }^{345,346}$. The European Neuroendocrine Tumour Society (ENETS) consensus guidelines indicate that these patients should receive aggressive CRS in high-volume centres if complete resection can be achieved ${ }^{347}$; the role of HIPEC in this indication is not clear ${ }^{348}$. In the PSOGI analysis, $40 \%$ of 127 patients treated with CRS and HIPEC had 5-year overall survival $^{333}$.

\section{Quality of life}

The well-being and QoL of patients with cancer is determined by a complex interplay of disease-related and treatment-related effects on somatic and psychological symptoms and functioning. Oncological treatments can have positive or negative effects on QoL and this balance tends to shift over time. QoL should therefore be regarded as a longitudinal measure ${ }^{349-351}$.

Peritoneal metastases are more frequently symptomatic than metastases at other sites and abdominal pain, nausea and ascites can have profound negative effects on $\mathrm{QoL}^{352}$. In untreated patients, disease tends to progress rapidly with aggravation of symptoms and a dramatic decline in QoL especially during the last 3 months of life $^{353}$. In this context, bowel obstruction deserves particular mention, as physical and psychological suffering accompanies loss of essential functions of living as

\section{Box 2 | Patient experience}

My husband and I have been married for 61 years. We only had one daughter because of our busy professional lives. When we retired, we started travelling Europe in our camping car, enjoying each other's company. He was a veteran of war with some old injuries and under treatment for a severe heart condition from the age of 64 . All these did not prevent us from living well.

When the diagnosis of appendiceal adenocarcinoma with mucinous peritoneal metastases came, he was 80 and most of the big centres in our region refused to treat him. They dismissed him with little hope because of his age, heart problems and advanced disease. We eventually moved further away for a third opinion, while his status was altering as he could only walk with a cane. When PIPAC [post-pressurized intraperitoneal aerosol chemotherapy] was proposed, we answered 'yes' right away. All we asked for was another couple of years together, watching our grandson growing a little older.

After the first two PIPAC, his general status improved dramatically and we could return to an almost normal life. In total, he underwent 15 PIPAC and, in the time intervals between them, we visited France and Spain in our camping car sharing many joyful moments. He was able to honour umpire invitations for a French National Competition. We also spent time with the entire family and friends.

When PIPAC stopped being an option due to non-access, we still had some time left to be grateful for having each other and to prepare the last moments. He died quietly a couple of days after our 61st wedding anniversary, 3 years after the diagnosis of an end-stage disease.

This statement was provided by Jeanine, the wife of a patient with peritoneal surface malignancy. well as lack of treatment options and consequent loss of hope $\mathrm{h}^{350}$.

Systemic chemotherapy remains the standard treatment for metastatic disease. While survival benefits remain modest for peritoneal metastases compared with metastases at other sites, systemic chemotherapy can have a profound negative effect on QoL, particularly in patients with a good performance status ${ }^{354,355}$. A close partnership between doctors, patients and their families with transparent and honest information on expected benefits, potential risks and treatment options is therefore of utmost importance to define the optimal treatment for the individual patient by shared decision-making. Frequently, there is already a profound misunderstanding between patients and care providers concerning the intent of treatment and prognosis ${ }^{356-358}$. Although patients with potentially curable disease are more likely to accept treatment-related adverse effects with effects on QoL and functioning, priorities and expectations might be very different in the palliative setting. Indeed, QoL and patient-related outcome and experience measures are increasingly used in routine clinical practice and as primary outcomes in research in the palliative setting. Several tools are available to assess these outcomes but none of them is specific for patients with $\mathrm{PSM}^{351}$. Thus, ongoing international efforts concentrate on the creation of dedicated tools to measure QoL and patient-related outcome measures specifically for patients with PSM. These tools will have to be validated in different countries to account for socio-cultural diversity ${ }^{359,360}$.

CRS combined with HIPEC is performed in most patients with a curative intent. With a potential for cure and long-term survival, a high risk of perioperative morbidity and mortality seems acceptable $\mathrm{e}^{6,45,361-363}$. In addition, patients have to be aware of a transitory deterioration of QoL lasting 6 months after surgery before getting back to baseline performance and surpassing QoL and symptom scores of patients undergoing systemic palliative chemotherapy ${ }^{364-366}$.

In the palliative setting and in patients with limited life expectancy, QoL gains more importance when evaluating treatment options. PIPAC has been shown to be a safe and feasible treatment option in patients with therapy-refractory disease who are not candidates for a potentially curative approach ${ }^{3,353}$. In this desperate setting, about two-thirds of patients will have an objective treatment response with no negative effect on QoL. Symptoms improve in $>50 \%$ of repeatedly treated patients who can gain additional quality lifetime and hope $^{3,350,353}$ (BOX 2).

\section{Outlook}

\section{Personalized medicine}

Organoids are a 3D cell culture method using patient tissues to create a personalized tumour model to study patient-specific characteristics ${ }^{367}$. Patient-derived organoids to test chemosensitivity and predict treatment resistance and response have been explored ${ }^{368,369}$, including for colorectal PSM ${ }^{145}$. However, more efficient models to grow organoids need to be developed. This methodology is a promising approach to personalized 
intraperitoneal therapy but the clonal pressures and considerable heterogeneity that occur during therapy are substantial barriers to widespread adoption.

In addition, other models for testing chemosensitivity have been developed over the past few years, including xenograft, 2D cell monolayer, and 3D sphere and 3D ex vivo tumour models. In one study, chemosensitivity evaluated with $3 \mathrm{D}$ ex vivo models correlated more accurately with the response to chemotherapy in in vivo mouse models than the other models ${ }^{370}$.

\section{Nanomedicines for intraperitoneal therapy}

Major drawbacks of intraperitoneal therapies are the rapid clearance of chemotherapeutics from the peritoneal cavity to the systemic circulation ${ }^{371}$ and low tumour-targeting specificity. Nanomedicines (nanoparticles of 1-1,000 nm size) are widely used as delivery vehicles for therapeutic molecules, such as small molecules, proteins or nucleic acids, and are a promising platform when applied via different routes. For example, nanoparticle albumin-bound (nab) paclitaxel and liposomal doxorubicin are approved for intravenous use in clinical oncology and nucleic acid-based nanomedicines, such as coronavirus disease 2019 (COVID-19) vaccines, are administered intramuscularly ${ }^{372,373}$. Paclitaxel is a hydrophobic chemotherapy compound with a high molecular weight that has characteristic retention within the peritoneal space following intraperitoneal administration, making it an attractive molecule for the treatment of gastric PSM ${ }^{374}$.

In the past decade, the intraperitoneal use of nanomedicines has received increasing attention. Several studies have demonstrated benefits of intraperitoneal delivery, particularly for nucleic acids ${ }^{375-377}$; however, rapid clearance remains an unsolved problem. Sustained-release or depot systems loaded with nanoparticles or applying nanomedicines using the PIPAC technology $y^{378-380}$ have shown promising results in animal models but clinical data are lacking. The difficulty in the first strategy lies in the large surface area of the peritoneum and the need for homogenous distribution of the nanotherapeutics while preventing adhesion to tissues that may lead to inflammation ${ }^{381,382}$. The second strategy offers the advantage of uniform distribution of the medication in the peritoneum but it is unclear whether tumour killing is as effective as that of chemotherapeutics in humans and whether it can be applied to using nucleic acid agents.

Alternatively, the residence time of intraperitoneal drugs may be prolonged by incorporation in injectable depots and hydrogels, which enable loading conventional chemotherapy or nanoparticles in an entangled polymer network ${ }^{383,384}$. In addition to their potential to control drug release, certain hydrogels have the benefit of preventing postsurgical peritoneal adhesion formation $^{385}$. Other biomaterials for intraperitoneal drug delivery include sustained-release implantable matrices and nanotextiles ${ }^{386,387}$. These slow-release platforms enable a metronomic dosing strategy, which enhances antitumour efficacy with minimal systemic toxicity ${ }^{387}$. Clinical studies are awaited to establish their utility in patients with PSM.
Oncolytic viruses are highly versatile therapeutic platforms that can be genetically engineered to provide targeted anticancer and/or immune-modulating effects. Advantages of oncolytic viruses include selective replication in tumour cells, induction of immunogenic cell death and activation of immune responses ${ }^{388}$. The effects of oncolytic virus therapy on the tumour microenvironment enables synergism with immune-checkpoint inhibitors $^{389}$. Several studies have investigated the use of intraperitoneal delivery of oncolytic viruses in animal models of PSM and results from the first trials in humans are already available ${ }^{390}$. Intraperitoneal oncolytic vaccinia virus expressing an IL-15-IL-15Ra complex increased cytotoxic function of $\mathrm{CD}^{+} \mathrm{T}$ cells and improved survival in a mouse colorectal PSM model ${ }^{391}$. Another study in a colorectal PSM mouse model found that intraperitoneal delivery of vaccinia virus encoding murine GM-CSF activated dendritic cells and $\mathrm{CD}^{+}$ $\mathrm{T}$ cells, resulting in synergistic action when combined with immune-checkpoint inhibitors ${ }^{392}$. Clearly, oncolytic virus approaches hold promise in the treatment of patients with PSM.

\section{Surgical innovation}

Malignant disease can remain in the peritoneum at the end of a supposedly CC-0 CRS despite a macroscopically normal looking peritoneum. Up to $27.2 \%, 12.2 \%$ and $26.6-50 \%$ of patients with PSM of ovarian cancer, appendiceal cancer or mesothelioma origin, respectively, had malignancy in randomly selected peritoneal biopsy samples ${ }^{393}$. Thus, tools to achieve a more precise peritonectomy must be developed.

Near-infrared, fluorescence-guided surgery has great potential in the field of PSM. Some of the most popular uses of this technique are to assess bowel anastomosis perfusion or for sentinel node navigation ${ }^{394}$, but one of the most innovative and promising uses is the real-time detection of cancerous tissue using targeted or 'smart' fluorescent dyes. In addition to indocyanine green (the most commonly used fluorophore), which was shown to increase detection of PSM by up to $30 \%$ in patients with colorectal cancer ${ }^{395}$, fluorescence-guided surgery using targeted dyes has the potential to become routine to optimize $\mathrm{CRS}^{396}$. For example, use of a fluorescent dye targeting FRA, which is overexpressed in up to $95 \%$ of epithelial ovarian cancers, improved the number of tumour nodules detected by surgeons almost fivefold compared with standard observation ${ }^{397}$. So-called smart dyes are now being tested, including new tumour-targeted near-infrared dyes that may enable quicker, deeper and stronger imaging applications ${ }^{396}$.

Robotic peritonectomy has rarely been reported despite the advantages that robotic surgery offers in accomplishing complex abdominal procedures, and a complete, standardized description of robotic peritonectomy is not available so far, only reports of partial peritonectomies $^{398,399}$.

Whether patients with non-resectable PSM from an aggressive primary tumour or no response or progression despite PIPAC treatment could benefit from ePIPAC (electrostatic precipitation PIPAC) ${ }^{400}$ is currently being investigated. Deeper penetration of the 
chemotherapy has been observed but whether this correlates with an increased response rate or improvement in survival remains unclear ${ }^{401}$. Other technologies such as hPIPAC (hyperthermic PIPAC) are currently at early stages of research ${ }^{402}$.

\section{Challenge of trials in surgical oncology}

The design of clinical studies to evaluate the efficacy of surgery in patients with PSM is hampered by considerable heterogeneity. PSM can have various origins and, among the most common causes, such as colorectal and ovarian cancer, specific genetic and molecular landscapes can affect treatment response. In addition, the outcome of clinical trials that include a study group with complex surgery might be substantially affected by bias that is difficult to control, including variability in skill, experience, surgical technique and methods of adjuvant intraperitoneal drug delivery or HIPEC ${ }^{403,404}$.

In addition, in phase III randomized oncology trials, overall survival is commonly regarded as the optimal hard end point because of its undisputed significance and precision of measurement. However, in surgical oncology trials, its importance is affected by frequent crossover, long accrual times for cancers that are less lethal, and by subsequent therapy in patients who often have survival periods of years after initial surgery and receive multiple additional systemic treatments. In patients with PSM, progression-free survival is the preferred trial end point, either as a surrogate of overall survival if progression-free survival has been proven to correlate with overall survival, or because of the clinical benefit of preventing or delaying peritoneal disease recurrence, which is known to cause potentially serious morbidity. Disadvantages of the use of progression-free survival include the difficulty to timely and accurately diagnose recurrent peritoneal disease and that it does not always correlate with overall survival ${ }^{405}$.

The understanding, investigation and treatment of primary and metastatic PSM has greatly improved in the past few years and further exciting developments are expected. However, challenges remain. It is important not only to offer the best treatment option and develop intraperitoneal therapies that live up to the quality of current systemic therapies but also to define the optimal treatment sequence according to primary tumour, disease extent and patient preferences. New imaging modalities, less invasive surgery, nanomedicines and targeted therapies are the basis on which a new era of intraperitoneal therapy is being built, which will bring long-term improvements in patient outcomes.

Published online: 16 December 2021
1. Sadeghi, B. et al. Peritoneal carcinomatosis from nongynecologic malignancies: results of the EVOCAPE 1 multicentric prospective study. Cancer $\mathbf{8 8}, 358-363$ (2000).

2. Sugarbaker, P. H. Peritonectomy procedures. Ann. Surg. 221, 29-42 (1995).

3. Alyami, M. et al. Pressurised intraperitoneal aerosol chemotherapy: rationale, evidence, and potential indications. Lancet Oncol. 20, e368-e377 (2019). Comprehensive evidence-based overview on the PIPAC technique for PSM of various origins and its feasibility, safety and efficacy, including a summary of current indications for PIPAC and HIPEC treatment.

4. Glehen, O. et al. Toward curative treatment of peritoneal carcinomatosis from nonovarian origin by cytoreductive surgery combined with perioperative intraperitoneal chemotherapy: a multi-institutional study of 1,290 patients. Cancer 116, 5608-5618 (2010).

French multicentre study that gives an excellent overview on outcomes of CRS and HIPEC for patients with PSM of gastrointestinal origin. In addition, risk factors for poor outcomes are presented.

5. Van der Speeten, K., Lemoine, L. \& Sugarbaker, P. Overview of the optimal perioperative intraperitoneal chemotherapy regimens used in current clinical practice. Pleura Peritoneum. 2, 63-72 (2017).

6. Foster, J. M. et al. Morbidity and mortality rates following cytoreductive surgery combined with hyperthermic intraperitoneal chemotherapy compared with other high-risk surgical oncology procedures. JAMA Netw. Open 2, e186847 (2019).

7. Hübner, M. et al. Guidelines for perioperative care in cytoreductive surgery (CRS) with or without hyperthermic intraperitoneal chemotherapy (HIPEC): Enhanced recovery after surgery (ERAS ) Society Recommendations - part I: preoperative and intraoperative management. Eur. J. Surg. Oncol. 46, 2292-2310 (2020).

Evidence-based Delphi consensus for optima perioperative care for patients undergoing CRS \pm HIPEC: ERAS guidelines.

8. Cortês-Guiral, D., Mohamed, F., Glehen, O \& Passot, G. Prehabilitation of patients undergoing cytoreductive surgery (CRS) and hyperthermic intraperitoneal chemotherapy (HIPEC) for peritoneal malignancy. Eur. J. Surg. Oncol. 47, 60-64 (2021).

9. Hübner, M. et al. Guidelines for Perioperative Care in Cytoreductive Surgery (CRS) with or without hyperthermic IntraPEritoneal chemotherapy (HIPEC): Enhanced Recovery After Surgery (ERAS ${ }^{\circledR}$ ) Society Recommendations - part II: postoperative management and special considerations. Eur. J. Surg. Oncol. 46, 2311-2323 (2020)

10. Coleridge, S. L. et al. Chemotherapy versus surgery for initial treatment in advanced ovarian epithelial cancer. Cochrane Database Syst. Rev. 2019, CD005343 (2019).

11. Ruscito, l. et al. Incorporating parp-inhibitors in primary and recurrent ovarian cancer: a meta-analysis of 12 phase II/III randomized controlled trials. Cancer Treat. Rev. 87, 102040 (2020)

12. Goere, D. et al. Is there a possibility of a cure in patients with colorectal peritoneal carcinomatosis amenable to complete cytoreductive surgery and intraperitoneal chemotherapy? Ann. Surg. 257 1065-1071 (2013)

13. Jaime, P. FIGO Committee on Gynecologic Oncology FIGO's staging classification for cancer of the ovary, fallopian tube, and peritoneum: abridged republication. J. Gynecol. Oncol. 26, 87-89 (2015).

14. Sung, H. et al. Global cancer statistics 2020 GLOBOCAN estimates of incidence and mortality worldwide for 36 cancers in 185 countries. CA Cancer J. Clin. 71, 209-249 (2021).

15. Bloemendaal, A., Verwaal, V., Van Ruth, S., Boot, H. $\&$ Zoetmulder, F. Conventional surgery and systemic chemotherapy for peritoneal carcinomatosis of colorectal origin: a prospective study. Eur. J. Surg. Oncol. 31, 1145-1151 (2005)

16. Chu, D. Z., Lang, N. P., Thompson, C. Osteen, P. K $\&$ Westbrook, K. C. Peritioneal carcinomatosis in nongynecologic malignancy. A prospective study of prognostic factors. Cancer 63, 364-367 (1989).

17. Klos, D et al. Trends in peritoneal surface malignancies: evidence from a Czech nationwide population-based study. World J. Surg. Oncol. 17 182 (2019).

Epidemiology of PM of ovarian origin as the most frequently concerned tumour entity.

18. Zhang, Y. et al. Global patterns and trends in ovarian cancer incidence: age, period and birth cohort analysis. BMC Cancer 19, 984 (2019).

19. Torre, L. A. et al. Ovarian cancer statistics, 2018. CA Cancer J. Clin. 68, 284-296 (2018)

20. Burg, L. et al. Incidence and predictors of peritonea metastases of gynecological origin: a populationbased study in the Netherlands. J. Gynecol. Oncol. 31, e58 (2020).
21. Bray, F. Loos, A. H. Tognazzo, S. \& La Vecchia, C. Ovarian cancer in Europe: cross-sectional trends in incidence and mortality in 28 countries, 1953-2000. Int. J. Cancer 113, 977-990 (2005)

22. National Academies of Sciences, Engineering, and Medicine. Ovarian Cancers: Evolving Paradigms in Research and Care (National Academies Press; 2016).

23. Abbasi, S. Y., El Taani, H., Saad, A. \& Badheeb, A Advanced gastric cancer in Jordan from 2004 to 2008: a study of epidemiology and outcomes. Gastrointest. Cancer Res. 4, 122 (2011).

24. Thomassen, I. et al. Peritoneal carcinomatosis of gastric origin: a population-based study on incidence, survival and risk factors. Int. J. Cancer 134, 622-628 (2014).

25. Rijken, A. et al. The burden of peritoneal metastases from gastric cancer: a systematic review on the incidence, risk factors and survival. J. Clin. Med. 10, 4882 (2021).

26. Segelman, J. et al. Incidence, prevalence and risk factors for peritoneal carcinomatosis from colorectal cancer. Br. J. Surg. 99, 699-705 (2012).

27. Jayne, D., Fook, S., Loi, C. \& Seow-Choen, F. Peritoneal carcinomatosis from colorectal cancer. J. Br. Surg. 89, 1545-1550 (2002).

28. Van den Heuvel, M., Lemmens, V., Verhoeven, R. $\&$ de Hingh, I. The incidence of mucinous appendiceal malignancies: a population-based study. Int. J. Colorectal Dis. 28, 1307-1310 (2013)

29. Flanagan, M. et al. Peritoneal metastases from extra-abdominal cancer - a population-based study. Eur. J. Surg. Oncol. 44, 1811-1817 (2018)

30. Satoh, H. et al. Peritoneal carcinomatosis in lung cancer patients. Oncol. Rep. 8, 1305-1307 (2001).

31. Bertozzi, S. et al. Prevalence, risk factors, and prognosis of peritoneal metastasis from breast cancer. SpringerPlus 4, 1-8 (2015)

32. Cashin, P. H., Jansson Palmer, G., Asplund, D., Graf, W. \& Syk, I. Peritoneal mesothelioma in Sweden: a population-based study. Cancer Med. 8 6468-6475 (2019).

33. Consonni, D. et al. Peritoneal mesothelioma and asbestos exposure: a population-based case-control study in Lombardy, Italy. Occup. Environ. Med. 76 545-553 (2019).

34. Alpert, N., van Gerwen, M. \& Taioli, E. Epidemiology of mesothelioma in the 21 st century in Europe and the United States, 40 years after restricted/banned asbestos use. Transl. Lung Cancer Res. 9 (Suppl. 1), S28 (2020). 
35. Chua, T. C. et al. Early-and long-term outcome data of patients with pseudomyxoma peritonei from appendiceal origin treated by a strategy of cytoreductive surgery and hyperthermic intraperitoneal chemotherapy. J. Clin. Oncol. 30, 2449-2456 (2012).

36. Quénet, F. et al. Cytoreductive surgery plus hyperthermic intraperitoneal chemotherapy versus cytoreductive surgery alone for colorectal peritoneal metastases (PRODIGE 7): a multicentre, randomised, open-label, phase 3 trial. Lancet Oncol. 22, 256-266 (2021).

Pivotal RCT comparing the benefit of short duration oxaliplatin-based HIPEC in addition to CRS and perioperative chemotherapy for patients with colorectal PM.

37. Yan, T. D. et al. A novel tumor-node-metastasis (TNM) staging system of diffuse malignant peritoneal mesothelioma using outcome analysis of a multiinstitutional database. Cancer 117, 1855-1863 (2011)

38. Ishigami, H. et al. Phase III trial comparing intraperitoneal and intravenous paclitaxel plus S-1 versus cisplatin plus S-1 in patients with gastric cancer with peritoneal metastasis: PHOENIX-GC trial. J. Clin. Oncol. 36, 1922-1929 (2018). Landmark randomized controlled trial evaluating the impact of combined intravenous and intraperitoneal chemotherapy (neoadjuvant intraperitoneal and systemic chemotherapy) on the prognosis of patients with gastric PM.

39. Kitayama, J. et al. ASO author reflections: repeated intraperitoneal paclitaxel with systemic chemotherapy as the first-line treatment for peritoneal malignancy. Ann. Surg. Oncol. 28, 3871-3872 (2021)

40. Ishigami, H., Kitayama, J., Yamaguchi, H., Emoto, S $\&$ Watanabe, T. Phase II study of intravenous and intraperitoneal paclitaxel combined with S-1 for gastric cancer with metastases to the distant peritoneum. Ann. Oncol. 23, ix233 (2012)

41. Verwaal, V. J. et al. Randomized trial of cytoreduction and hyperthermic intraperitoneal chemotherapy versus systemic chemotherapy and palliative surgery in patients with peritoneal carcinomatosis of colorectal cancer. J. Clin. Oncol. 21, 3737-3743 (2003).

42. Noiret, B. et al. Centralization and oncologic training reduce postoperative morbidity and failure-to-rescue rates after cytoreductive surgery and hyperthermic intraperitoneal chemotherapy for peritoneal surface malignancies: study on a 10-year national French practice. Ann. Surg. 272, 847-854 (2020).

43. Schuitevoerder D., Sherman, S. K., Izquierdo, F. J. Eng, O. S. \& Turaga, K. K. Assessment of the surgical workforce pertaining to cytoreductive surgery and hyperthermic intraperitoneal chemotherapy in the United States. Ann. Surg. Oncol. 27, 3097-3102 (2020).

44. Gopinath, K. \& Bhatt, A. Preface 'Changing trend in peritoneal surface oncology in Asian countries'. Indian J. Surg. Oncol. 10, 1-2 (2019).

45. van Driel, W. J. et al. Hyperthermic intraperitoneal chemotherapy in ovarian cancer. N. Engl. J. Med. 378 230-240 (2018)

Dutch multicentre randomized controlled trial showing large survival benefit with no increase of morbidity in favour of adding cisplatin-based HIPEC to CRS for patients with ovarian cancer undergoing interval surgery after neoadjuvant chemotherapy.

46. Low, R. N., Barone, R. M. \& Rousset, P. Peritoneal MRI in patients undergoing cytoreductive surgery and HIPEC: history, clinical applications, and implementation. Eur. J. Surg. Oncol. 47, 65-74 (2021).

47. Franko, J. Therapeutic efficacy of systemic therapy for colorectal peritoneal carcinomatosis: surgeon's perspective. Pleura Peritoneum 3, 20180102 (2018).

48. Müller, H., Hotopp, T., Tofeili, A. \& Wutke, K. Systemic chemotherapy using FLOT-regimen combined with cytoreductive surgery plus HIPEC for treatment of peritoneal metastasized gastric cancer. Hepatogastroenterology 61, 703-706 (2014).

49 Becker O Beaulaton, C. Masliah-Planchon, J. Servois, V. \& Watson, S. Nivolumab activity in advanced refractory malignant peritoneal mesothelioma. Eur. J. Cancer 2020, S0959-S8049 (1990).

50. Glehen, O. et al. GASTRICHIP: D2 resection and hyperthermic intraperitoneal chemotherapy in locally advanced gastric carcinoma: a randomized and multicenter phase III study. BMC Cancer 14, 183 (2014)

51. Honoré, C. et al. Ninety percent of the adverse outcomes occur in $10 \%$ of patients: can we identify the populations at high risk of developing peritoneal metastases after curative surgery for colorectal cancer? Int. J. Hyperth. 33, 505-510 (2017).

52. Smeenk, R., Van Velthuysen, M., Verwaal, V. \& Zoetmulder, F. Appendiceal neoplasms and pseudomyxoma peritonei: a population based study. Eur. J. Surg. Oncol. 34, 196-201 (2008).

53. Yurgelun, M. B. et al. Cancer susceptibility gene mutations in individuals with colorectal cancer. J. Clin. Oncol. 35, 1086-1095 (2017).

54. Pietragalla, A., Arcieri, M., Marchetti, C., Scambia, G. \& Fagotti, A. Ovarian cancer predisposition beyond BRCA1 and BRCA2 genes. Int. J. Gynecol. Cancer 30 1803-1810 (2020)

55. Win, A. K. et al. Prevalence and penetrance of major genes and polygenes for colorectal cancer. Cancer Epidemiol. Biomark. Prev. 26, 404-412 (2017).

56. Corso, G. et al. Geographical distribution of E-cadherin germline mutations in the context of diffuse gastric cancer: a systematic review. Cancers 13, 1267 (2021).

57. Testa, J. R. et al. Germline BAP1 mutations predispose to malignant mesothelioma Nat. Genet. 43 1022-1025 (2011)

58. Foulkes, W. D. Inherited susceptibility to common cancers. N. Engl. J. Med. 359, 2143-2153 (2008)

59. Sekine M Nishino, K \& Enomoto, T BRCA genetic test and risk-reducing salpingo-oophorectomy for hereditary breast and ovarian cancer: state-of-the-art. Cancers 13, 2562 (2021)

60. Sinicrope, F. A. Lynch syndrome-associated colorectal cancer. N. Engl. J. Med. 379, 764-773 (2018).

61. Rustgi, S. D., Ching, C. K. \& Kastrinos, F. Inherited predisposition to gastric cancer. Gastrointest. Endosc. Clin. 31, 467-487 (2021)

62. Albanese, A. M. et al. Peritoneal surface area: measurements of 40 structures covered by peritoneum: correlation between total peritoneal surface area and the surface calculated by formulas. Surg. Radiol. Anat. 31, 369-377 (2009).

63. Flessner, M. F. Peritoneal transport physiology: insights from basic research. J. Am. Soc. Nephrol. 2, 122-135 (1991).

64. Azuar, A. S. et al. Impact of surgical peritoneal environment on postoperative tumor growth and dissemination in a preimplanted tumor model. Surg. Endosc. 23, 1733-1739 (2009).

65. Binda, M. M. Molinas, C. R., Hansen, P. \& Koninckx, P. R. Effect of desiccation and temperature during laparoscopy on adhesion formation in mice. Fertil. Steril. 86, 166-175 (2006).

66. Carpinteri, S. et al. Peritoneal tumorigenesis and inflammation are ameliorated by humidified-warm carbon dioxide insufflation in the mouse. Ann. Surg. Oncol. 22 (Suppl. 3), S1540-S1547 (2015).

67. Mutsaers, S. E., Whitaker, D. \& Papadimitriou, J. M Changes in the concentration of microvilli on the fre surface of healing mesothelium are associated with alterations in surface membrane charge. J. Pathol. 180, 333-339 (1996)

68. Sampurno, S. et al. Modes of carbon dioxide delivery during laparoscopy generate distinct differences in peritoneal damage and hypoxia in a porcine model. Surg. Endosc. 34, 4395-4402 (2020).

69. Volz, J., Koster, S., Spacek, Z. \& Paweletz, N. Characteristic alterations of the peritoneum after carbon dioxide pneumoperitoneum. Surg. Endosc. 13 611-614 (1999)

70. Mutsaers, S. E., Prele, C. M., Pengelly, S. \& Herrick, S. E. Mesothelial cells and peritoneal homeostasis. Fertil. Steril. 106, 1018-1024 (2016).

71. LaRocca, P. J. \& Rheinwald, J. G. Coexpression of simple epithelial keratins and vimentin by human mesothelium and mesothelioma in vivo and in culture. Cancer Res. 44, 2991-2999 (1984).

72. Dobbie, J. W., Pavlina, T., Lloyd, J. \& Johnson, R. C. Phosphatidylcholine synthesis by peritoneal mesothelium: its implications for peritoneal dialysis. Am. J. Kidney Dis. 12, 31-36 (1988) Comprehensive science-based overview on the physiology of the normal and diseased peritoneum an orphan organ.

73. Grupp, A. et al. The expression patterns of peritoneal defensins. Perit. Dial. Int. 27, 654-662 (2007)

74. Tang, S. et al. Regulation of complement C3 and C4 synthesis in human peritoneal mesothelial cells by peritoneal dialysis fluid. Clin. Exp. Immunol. 136 85-94 (2004)

75. Zarrinkalam, K. H., Leavesley, D. I. Stanley, J. M. Atkins, G. J. \& Faull, R. J. Expression of defensin antimicrobial peptides in the peritoneal cavity of patients on peritoneal dialysis. Perit. Dial. Int. $\mathbf{2 1}$ 501-508 (2001).
76. van Baal, J. O. et al. The histophysiology and pathophysiology of the peritoneum. Tissue Cell 49 95-105 (2017)

77. Yamaji, K. et al. Interleukin- 6 production by peritoneal mesothelial cells and its regulation by inflammatory factors in rats administered carbon tetrachloride intraperitoneally. Toxicol. Appl. Pharmacol. 226, 38-45 (2008)

78. Jonjic, N. et al. Expression of adhesion molecules and chemotactic cytokines in cultured human mesothelial cells. J. Exp. Med. 176, 1165-1174 (1992).

79. Yao, V., Platell, C. \& Hall, J. C. Peritoneal mesothelial cells produce inflammatory related cytokines. Anz. J. Surg. 74, 997-1002 (2004)

80. Colmont, C. S. et al. Human peritoneal mesothelial cells respond to bacterial ligands through a specific subset of Toll-like receptors. Nephrol. Dial. Transplant. 26, 4079-4090 (2011).

81. Hausmann, M. J., Rogachev, B., Weiler, M. Chaimovitz, C. \& Douvdevani, A. Accessory role of human peritoneal mesothelial cells in antigen presentation and T-cell growth. Kidney Int. 57, 476-486 (2000)

82. Park, J. H. et al. Nod $1 /$ RICK and TLR signaling regulate chemokine and antimicrobial innate immune responses in mesothelial cells. J. Immunol. 179 , 514-521 (2007).

83. Foussat, A. et al. Production of stromal cell-derived factor 1 by mesothelial cells and effects of this chemokine on peritoneal B lymphocytes. Eur. J. Immunol. 31, 350-359 (2001).

84. Kato, S. et al. Endotoxin-induced chemokine expression in murine peritoneal mesothelial cells: the role of toll-like receptor 4. J. Am. Soc. Nephrol. 15, 1289-1299 (2004).

85. Boulanger, E. et al. AGEs bind to mesothelial cells via RAGE and stimulate VCAM-1 expression. Kidney Int. 61, 148-156 (2002)

86. Cui, L. et al. Biodefense function of omental milky spots through cell adhesion molecules and leukocyte proliferation. Cell Tissue Res. 310, 321-330 (2002).

87. Liang Y $Y$ \& Sasaki, K. Expression of adhesion molecules relevant to leukocyte migration on the microvilli of liver peritoneal mesothelial cells. Anat. Rec. 258, 39-46 (2000).

88. Valle, M. T. et al. Antigen-presenting function of human peritoneum mesothelial cells. Clin. Exp. Immunol. 101 172-176 (1995)

89. Shaw, T. J. et al. Human peritoneal mesothelial cells display phagocytic and antigen-presenting functions to contribute to intraperitoneal immunity. Int. J. Gynecol. Cancer 26, 833-838 (2016).

90. Bellingan, G. J. et al. Adhesion molecule-dependent mechanisms regulate the rate of macrophage clearance during the resolution of peritoneal inflammation. J. Exp. Med. 196, 1515-1521 (2002).

91. Zsiros, V. \& Kiss, A. L. Cellular and molecular events of inflammation induced transdifferentiation (EMT) and regeneration (MET) in mesenteric mesothelial cells. Inflamm. Res. 69, 1173-1179 (2020).

92. Wilson, R. B., Archid, R. \& Reymond, M. A Reprogramming of mesothelial-mesenchymal transition in chronic peritoneal diseases by estrogen receptor modulation and TGF-beta 1 inhibition. Int. J. Mol. Sci. 21, 4158 (2020)

93. Pronk, A. et al. Thrombogenicity and procoagulant activity of human mesothelial cells. Arterioscler. Thromb. 12, 1428-1436 (1992).

94. lakhiaev, A. \& Idell, S. Activation and degradation of protein $C$ by primary rabbit pleural mesothelial cells. Lung 184, 81-88 (2006).

95. Schaefer, B. et al. Quantitative histomorphometry of the healthy peritoneum. Sci. Rep. 6, 21344 (2016).

96. Owens, M. W. \& Grimes, S. R. Pleural mesothelial cell response to inflammation: tumor necrosis factorinduced mitogenesis and collagen synthesis. Am. J. Physiol. 265, L382-L388 (1993).

97. Yang, W. S., Kim, B. S., Lee, S. K., Park, J. S. \& Kim, S. B. Interleukin-1 beta stimulates the production of extracellular matrix in cultured human peritoneal mesothelial cells. Perit. Dial. Int. 19, 211-220 (1999).

98. Owens, M. W. \& Milligan, S. A. Growth factor modulation of rat pleural mesothelial cell mitogenesis and collagen synthesis. Effects of epidermal growth factor and platelet-derived factoR. Inflammation 18, 77-87 (1994).

99. Saed, G. M., Zhang, W., Chegini, N., Holmdahl, L. \& Diamond, M. P. Alteration of type I and III collagen expression in human peritoneal mesothelial cells in response to hypoxia and transforming growth factor-beta1. Wound Repair. Regen. 7, 504-510 (1999). 
100. Rennard, S. I. et al. Role of pleural mesothelial cells in the production of the submesothelial connective tissue matrix of lung. Am. Rev. Respir. Dis. 130, 267-274 (1984).

101. Torres, K. et al. TGF-beta and inflammatory blood markers in prediction of intraperitoneal adhesions. Adv. Med. Sci. 63, 220-223 (2018)

102. Selgas, R. et al. Epithelial-to-mesenchymal transition of the mesothelial cell-its role in the response of the peritoneum to dialysis. Nephrol. Dial. Transplant. 21 (Suppl. 2), ii2-ii7 (2006).

103. Saed, G. M., Zhang, W., Chegini, N., Holmdahl, L. ¿ Diamond, M. P. Transforming growth factor beta isoforms production by human peritoneal mesothelial cells after exposure to hypoxia. Am. J. Reprod. Immunol. 43, 285-291 (2000).

104. Xiao, L, Sun, L, Liu, F Y Peng, Y M \& Duan, S. B Connective tissue growth factor knockdown attenuated matrix protein production and vascular endothelial growth factor expression induced by transforming growth factor-beta 1 in cultured human peritoneal mesothelial cells. Ther. Apher. Dial. 14, 27-34 (2010).

105. McArdle, C. S., McMillan, D. C. \& Hole, D. J. The impact of blood loss, obstruction and perforation on survival in patients undergoing curative resection for colon cancer. Br. J. Surg. 93, 483-488 (2006).

106. Komatsu, S. et al. Prognostic factors and scoring system for survival in colonic perforation. Hepatogastroenterology 52, 761-764 (2005).

107. Chen, H. S. \& Sheen-Chen, S. M. Obstruction and perforation in colorectal adenocarcinoma: an analysis of prognosis and current trends. Surgery 127 , 370-376 (2000)

108. Cheynel, N. et al. Incidence, patterns of failure, and prognosis of perforated colorectal cancers in a welldefined population. Dis. Colon. Rectum 52, 406-411 (2009).

109. Terauchi, M. et al. Possible involvement of TWIST in enhanced peritoneal metastasis of epithelial ovarian carcinoma. Clin. Exp. Metastasis 24, 329-339 (2007).

110. Kokenyesi, R., Murray, K. P., Benshushan, A Huntley, E. D. \& Kao, M. S. Invasion of interstitial matrix by a novel cell line from primary peritoneal carcinosarcoma, and by established ovarian carcinoma cell lines: role of cell-matrix adhesion molecules, proteinases, and E-cadherin expression. Gynecol. Oncol. 89, 60-72 (2003).

111. Christou, N. et al. E-cadherin: a potential biomarker of colorectal cancer prognosis. Oncol. Lett. 13, 4571-4576 (2017)

112. Hayashi, K. et al. Real-time Imaging of tumor-cell shedding and trafficking in lymphatic channels. Cancer Res. 67, 8223-8228 (2007).

113. Rutz, H. P. Hydrodynamic consequences of glycolysis - thermodynamic basis and clinical relevance. Cancer Biol. Ther. 3, 812-815 (2004).

114. Heldin, C. H., Rubin, K., Pietras, K. \& Ostman, A. High interstitial fluid pressure - an obstacle in cancer therapy. Nat. Rev. Cancer 4, 806-813 (2004).

115. Hansen, E. et al. Tumor-cells in blood shed from the surgical field. Arch. Surg. 130, 387-393 (1995).

116. Tanaka, N., Nobori, M. \& Suzuki, Y. Does bile spillage during an operation present a risk for peritoneal metastasis in bile duct carcinoma? Surg. Today 27, 1010-1014 (1997)

117. Meyers, M. A. Distribution of intraabdominal malignant seeding - dependency on dynamics of flow of ascitic fluid. Am. J. Roentgenol. 119, 198-206 (1973).

118. Lindberg, U., Karlsson, R., Lassing, I., Schutt, C. E. \& Hoglund, A. S. The microfilament system and malignancy. Semin. Cancer Biol. 18, 2-11 (2008)

119. Moffitt, L., Karimnia, N., Stephens, A. \& Bilandzic, M. Therapeutic targeting of collective invasion in ovarian cancer. Int. J. Mol. Sci. 20, 1466 (2019).

120. Zajac, O. et al. Tumour spheres with inverted polarity drive the formation of peritoneal metastases in patients with hypermethylated colorectal carcinomas. Nat. Cell Biol. 20, 296-306 (2018)

121. Bittinger, $F$. et al. PECAM-1 expression in human mesothelial cells: an in vitro study. Pathobiology 64 320-327 (1996)

122. Ziprin, P., Alkhamesi, N. A., Ridgway, P. F., Peck, D. H. \& Darzi, A. W. Tumour-expressed CD43 (sialophorin) mediates tumour-mesothelial cell adhesion. Biol. Chem. 385, 755-761 (2004).

123. Hassan, A. A., Artemenko, M., Tang, M. K. S. $\&$ Wong, A. S. T. Selectins: an important family of glycan-binding cell adhesion molecules in ovarian cancer. Cancers 12, 2238 (2020).
124. Gebauer, F. et al. Selectin binding is essential for peritoneal carcinomatosis in a xenograft model of human pancreatic adenocarcinoma in $\mathrm{pfp}^{-} / \mathrm{rag}^{-}$mice Gut 62, 741-750 (2013)

125. Borsig, L. et al. Selectin blocking activity of a fucosylated chondroitin sulfate glycosaminoglycan from sea cucumber. Effect on tumor metastasis and neutrophil recruitment. J. Biol. Chem. 282 14984-14991 (2007).

126. Eustache, J. H. et al. Wide net on surgery: the central role of neutrophil extracellular traps. Ann. Surg. 272 277-283 (2020)

127. Oosterling, S. J. et al. Anti-beta 1 integrin antibody reduces surgery-induced adhesion of colon carcinoma cells to traumatized peritoneal surfaces. Ann. Surg. 247, 85-94 (2008).

128. Ween, M. P. Oehler, M. K. \& Ricciardelli, C. Role of versican, hyaluronan and CD44 in ovarian cancer metastasis. Int. J. Mol. Sci. 12, 1009-1029 (2011).

129. Hilliard, T. S. The impact of mesothelin in the ovarian cancer tumor microenvironment. Cancers 10, 277 (2018).

130. Koppe, M. J., Nagtegaal, I. D., de Wilt, J. H. \& Ceelen, W. P. Recent insights into the pathophysiology of omental metastases. J. Surg. Oncol. 110, 670-675 (2014).

131. Gerber, S. A et al. Preferential attachment of peritoneal tumor metastases to omental immune aggregates and possible role of a unique vascular microenvironment in metastatic survival and growth. Am. J. Pathol. 169, 1739-1752 (2006).

132. Nieman, K. M. et al. Adipocytes promote ovarian cancer metastasis and provide energy for rapid tumor growth. Nat. Med. 17, 1498-1503 (2011).

133. Meza-Perez, S. \& Randall, T. D. Immunological functions of the omentum. Trends Immunol. 38 526-536 (2017)

134. Sorensen, E. W. et al. Omental immune aggregates and tumor metastasis within the peritoneal cavity. Immunol. Res. 45, 185-194 (2009).

135. Zulfigar, M. et al. Krukenberg tumors: update on imaging and clinical features. Am. J. Roentgenol. 215 1020-1029 (2020)

136. van der Bij, G. J. et al. The perioperative period is an underutilized window of therapeutic opportunity in patients with colorectal cancer. Ann. Surg. 249, 727-734 (2009).

137. Klein, C. L. et al. Effects of cytokines on the expression of cell adhesion molecules by cultured human omenta mesothelial cells. Pathobiology 63, 204-212 (1995)

138. Ikari, Y., Yee, K. O. \& Schwartz, S. M. Role of alpha5beta 1 and alphavbeta3 integrins on smooth muscle cell spreading and migration in fibrin gels. Thromb. Haemost. 84, 701-705 (2000)

139. Basson, M. D. et al. Effects of increased ambient pressure on colon cancer cell adhesion. J. Cell. Biochem. 78, 47-61 (2000).

140. Ridgway, P. F. et al. Pneumoperitoneum augmented tumor invasiveness is abolished by matrix metalloproteinase blockade. Surg. Endosc. 16 533-536 (2002)

141. Jacobi, C. A. et al. Pneumoperitoneum with carbon dioxide stimulates growth of malignant colonic cells. Surgery 121, 72-78 (1997)

142. Heath, R. M., Jayne, D. G., O'Leary, R., Morrison, E. E. $\&$ Guillou, P. J. Tumour-induced apoptosis in human mesothelial cells: a mechanism of peritoneal invasion by Fas Ligand/Fas interaction. Br. J. Cancer $\mathbf{9 0}$, 1437-1442 (2004).

143. Carreiras, F. et al. Migration properties of the human ovarian adenocarcinoma cell line IGROV 1: importance of alpha $v$ beta 3 integrins and vitronectin. Int. J. Cancer 80, 285-294 (1999).

144. Lubbe, W. J. et al. Guanylyl cyclase c prevents colon cancer metastasis by regulating tumor epithelial cell matrix metalloproteinase-9. Cancer Res. 69 , 3529-3536 (2009).

145. Narasimhan, V. et al. Medium-throughput drug screening of patient-derived organoids from colorectal peritoneal metastases to direct personalized therapy. Clin. Cancer Res. 26, 3662-3670 (2020).

146. Wang Z. B. Li, M. \& Li, J. C. Recent advances in the research of lymphatic stomata. Anat. Rec. 293 754-761 (2020)

147. Li, J. C. \& Yu, S. M. Study on the ultrastructure of the peritoneal stomata in humans. Acta Anatomica. 141, 26-30 (1991).

148. Namba, Y. An electron microscopic demonstration of the invasion of tumor cells into the diaphragm [Japanese]. Nippon Geka Gakkai Zasshi. 90 1915-1921 (1989).
149. Yonemura, Y. et al. Trans-lymphatic metastasis in peritoneal dissemination. J. Gastrointest. Dig. Syst. S12, 007 (2013)

150. Li, Y. Y. \& Li, J. C. Cell signal transduction mechanism for nitric oxide regulating lymphatic stomata and its draining capability. Anat. Rec. 291, 216-223 (2008).

151. Nagy, J. A., Herzberg, K. T., Dvorak, J. M. \& Dvorak, H. F. Pathogenesis of malignant ascites formation: initiating events that lead to fluid accumulation. Cancer Res. 53, 2631-2643 (1993).

152. Anwar, A. \& Kasi, A. Peritoneal Cancer (StatPearls Publishing LLC, 2021).

153. Sugarbaker, P. H. Pictorial essays on peritoneal metastases imaging: CT, MRI and PET-CT (Nova Science Publishers, 2020).

154. Warde, P. et al. Computed tomography in advanced ovarian cancer: an evaluation of diagnostic accuracy. Am. J. Med. Sci. 293, 94-98 (1987).

155. Bhatt, A. et al. Prospective correlation of the radiological, surgical and pathological findings in patients undergoing cytoreductive surgery for colorectal peritoneal metastases: implications for the preoperative estimation of the peritoneal cancer index. Colorectal Dis. 22, 2123-2132 (2020).

156. Ahmed, S. A. et al. The accuracy of multi-detector computed tomography and laparoscopy in the prediction of peritoneal carcinomatosis index score in primary ovarian cancer. Acad. Radiol. 26 , 1650-1658 (2019).

157. Borley, J. et al. Radiological predictors of cytoreductive outcomes in patients with advanced ovarian cancer. BJOG 122, 843-849 (2015).

158. Sugarbaker, P. H. et al. Concerning CT features used to select patients for treatment of peritonea metastases, a pictoral essay. Int. J. Hyperth. 33, 497-504 (2017)

159. Gonzalez-Moreno, S., Gonzalez-Bayon, L., Ortega-Perez, G. \& Gonzalez-Hernando, C. Imaging of peritoneal carcinomatosis. Cancer J. 15, 184-189 (2009).

160. van 't Sant, I. et al. Diagnostic performance of imaging for the detection of peritoneal metastases: a meta-analysis. Eur. Radiol. 30, 3101-3112 (2020).

161. Koh, J. L., Yan, T. D., Glenn, D. \& Morris, D. L. Evaluation of preoperative computed tomography in estimating peritoneal cancer index in colorectal peritoneal carcinomatosis. Ann. Surg. Oncol. 16 327-333 (2009).

162. Dirisamer, A. et al. Detection of histologically proven peritoneal carcinomatosis with fused 18F-FDG-PET MDCT. Eur. J. Radiol. 69, 536-541 (2009).

163. Chandramohan, A., Thrower, A., Smith, S., Shah, N. \& Moran, B. "PAUSE": a method for communicating radiological extent of peritoneal malignancy. Clin. Radiol. 72, 972-980 (2017). Overview and comparison of available imaging modalities for PSM.

164. Li, J., Yan, R., Lei, J. \& Jiang, C. Comparison of PET with PET/CT in detecting peritoneal carcinomatosis: a meta-analysis. Abdom. Imaging 40, 2660-2666 (2015).

165. Wang W. et al Are positron emission tomographycomputed tomography (PET-CT) scans useful in preoperative assessment of patients with peritoneal disease before cytoreductive surgery (CRS) and hyperthermic intraperitoneal chemotherapy (HIPEC)? Int. J. Hyperth. 34, 524-531 (2018).

166. Dewulf, J., Adhikari, K., Vangestel, C., Wyngaert, T. V. D. \& Elvas, F. Development of antibody immuno-PET/ SPECT radiopharmaceuticals for imaging of oncological disorders-an update. Cancers 12, 1868 (2020).

167. Guzik, P et al. Identification of a PET radiotracer for imaging of the folate receptor- $\alpha$ : a potential tool to select patients for targeted tumor therapy. J. Nucl. Med. 62, 1475 (2021).

168. Low, R. N. \& Barone, R. M. Combined diffusionweighted and gadolinium-enhanced MRI can accurately predict the peritoneal cancer index preoperatively in patients being considered for cytoreductive surgical procedures. Ann. Surg. Oncol. 19, 1394-1401 (2012)

169. Dohan, A et al. Evaluation of the peritoneal carcinomatosis index with CT and MRI. Br. J. Surg. 104, 1244-1249 (2017)

170. Menassel, B. et al. Preoperative CT and MRI prediction of non-resectability in patients treated for pseudomyxoma peritonei from mucinous appendiceal neoplasms. Eur. J. Surg. Oncol. 42, 558-566 (2016).

171. Nougaret, S. et al. Radiomics and radiogenomics in ovarian cancer: a literature review. Abdom. Radiol. 46 2308-2322 (2021). 
172. Rodríguez-Enríquez, S. et al. Multi-biomarker pattern for tumor identification and prognosis. J. Cell. Biochem. 112, 2703-2715 (2011).

173. Carpelan-Holmström, M., Louhimo, J., Stenman, U. H., Alfthan, H. \& Haglund, C. CEA, CA 19-9 and CA 72-4 improve the diagnostic accuracy in gastrointestinal cancers. Anticancer Res. 22, 2311-2316 (2002).

174. Sturgeon, C. Practice guidelines for tumor marker use in the clinic. Clin. Chem. 48, 1151-1159 (2002).

175. Gebauer, G. \& Müller-Ruchholtz, W. Tumor marker concentrations in normal and malignant tissues of colorectal cancer patients and their prognostic relevance. Anticancer Res. 17, 2939-2942 (1997).

176. Emoto, S. et al. Clinical significance of CA125 and CA72-4 in gastric cancer with peritoneal dissemination. Gastric Cancer 15, 154-161 (2012).

177. Lai, I. R., Lee, W. J., Huang, M. T. \& Lin, H. H. Comparison of serum CA72-4, CEA, TPA, CA 19-9 and CA 125 levels in gastric cancer patients and correlation with recurrence. Hepatogastroenterology 49, 1157-1160 (2002).

178. Yamao, T. et al. Tumor markers CEA, CA19-9 and CA1 25 in monitoring of response to systemic chemotherapy in patients with advanced gastric cancer. Jpn. J. Clin. Oncol. 29, 550-555 (1999).

179. Moayyedi, P. et al. ACG and CAG clinical guideline: management of dyspepsia. Am. J. Gastroenterol. 112 988-1013 (2017).

180. Shaukat, A. et al. ACG clinical guidelines: colorectal cancer screening 2021. Am. J. Gastroenterol. 116, 458-479 (2021)

181. Montminy, E. M., Jang, A., Conner, M. \& Karlitz, J. J. Screening for colorectal cancer. Med. Clin. North. Am. 104, 1023-1036 (2020).

182. Zullo, A. et al. Diagnostic yield of upper endoscopy according to appropriateness: a systematic review. Dig. Liver Dis. 51, 335-339 (2019)

183. Frazzoni, L. et al. Systematic review with metaanalysis: the appropriateness of colonoscopy increases the probability of relevant findings and cancer while reducing unnecessary exams. Aliment. Pharmacol. Ther. 53, 22-32 (2021)

184. Raś, R. et al. Preoperative colonoscopy in patients with a supposed primary ovarian cancer. Medicine 98 e14929 (2019)

185. Valle, M., Federici, O. \& Garofalo, A. Patient selection for cytoreductive surgery and hyperthermic intraperitoneal chemotherapy, and role of laparoscopy in diagnosis, staging, and treatment. Surg. Oncol. Clin. North. Am. 21, 515-531 (2012).

186. Esquivel, J. et al. Cytoreductive surgery and hyperthermic intraperitoneal chemotherapy in the management of peritoneal surface malignancies of colonic origin: a consensus statement. Society of Surgical Oncology. Ann. Surg. Oncol. 14, 128-133 (2007).

187. Jacquet P. Sugarbaker P In Peritoneal Carcinomatosis: Principles of Management. Cancer Treatment and Research (ed. Sugarbaker P.) Vol. 82 359-374 (Springer US, 1996).

188. Patel, C. M., Sahdev, A. \& Reznek, R. H. CT, MRI and PET imaging in peritoneal malignancy. Cancer Imaging 11, 123-139 (2011).

189. Marmor, R. A., Kelly, K. J., Lowy, A. M. \& Baumgartner, J. M. Laparoscopy is safe and accurate to evaluate peritoneal surface metastasis prior to cytoreductive surgery. Ann. Surg. Oncol. 23. 1461-1467 (2016)

190. Pomel, C., Appleyard, T. L., Gouy, S., Rouzier, R. $\&$ Elias, D. The role of laparoscopy to evaluate candidates for complete cytoreduction of peritoneal carcinomatosis and hyperthermic intraperitoneal chemotherapy. Eur. J. Surg. Oncol. 31, 540-543 (2005).

191. von Breitenbuch, P., Boerner, T., Jeiter, T., Piso, P. \& Schlitt, H. J. Laparoscopy as a useful selection tool for patients with prior surgery and peritoneal metastases suitable for multimodality treatment strategies. Surg. Endosc. 32, 2288-2294 (2018).

192. Jayakrishnan, T. T. et al. Role of laparoscopy in patients with peritoneal metastases considered for cytoreductive surgery and hyperthermic intraperitoneal chemotherapy (HIPEC). World J. Surg. Oncol. 12, 270 (2014).

193. Iversen, L. H., Rasmussen, P. C. \& Laurberg, S. Value of laparoscopy before cytoreductive surgery and hyperthermic intraperitoneal chemotherapy for peritoneal carcinomatosis. Br. J. Surg. 100, 285-292 (2013).

194. van Oudheusden, T. R. et al. Peritoneal cancer patients not suitable for cytoreductive surgery and HIPEC during explorative surgery: risk factors, treatment options, and prognosis. Ann Surg. Oncol. 22, 1236-1242 (2015).

195. Passot, G. et al. Multicentre study of laparoscopic or open assessment of the peritoneal cancer index (BIG-RENAPE). Br. J. Surg. 105, 663-667 (2018).

196. von Renteln, D. et al. Standard diagnostic laparoscopy is superior to NOTES approaches: results of a blinded, randomized controlled porcine study. Endoscopy 44, 596-604 (2012)

197. Benzerdjeb, N. et al. Prognostic impact of combined progression index based on peritoneal grading regression score and peritoneal cytology in peritoneal metastasis. Histopathology 77, 548-559 (2020).

198. Delhorme, J. B. et al. Cytoreductive surgery and hyperthermic intraperitoneal chemotherapy for pseudomyxoma peritonei of appendicular and extraappendicular origin. Br. J. Surg. 105, 668-676 (2018).

199. Carr, N. J. et al. A consensus for classification and pathologic reporting of pseudomyxoma peritonei and associated appendiceal neoplasia: the results of the peritoneal surface Oncology Group International (PSOGI) Modified Delphi Process. Am. J. Surg. Pathol. 40, 14-26 (2016)

200. Ahadi, M., Sokolova, A., Brown, I., Chou, A. \& Gill, A. J. The 2019 World Health Organization Classification of appendiceal, colorectal and anal canal tumours: an update and critical assessment. Pathology 53 454-461 (2021)

201. Kusamura, S. et al. Peritoneal mesothelioma: PSOGI/ EURACAN clinical practice guidelines for diagnosis, treatment and follow-up. Eur. J. Surg. Oncol. 47. 36-59 (2021)

Comprehensive evidence-based guidelines and Delphi consensus on all aspects of care for patients having peritoneal mesothelioma.

202. Galateau Salle, F. et al. New insights on diagnostic reproducibility of biphasic mesotheliomas: a multiinstitutional evaluation by the international mesothelioma panel from the MESOPATH Reference Center. J. Thorac. Oncol. 13, 1189-1203 (2018)

203. Boeckx, N. et al. Primary tumor sidedness has an impact on prognosis and treatment outcome in metastatic colorectal cancer: results from two randomized first-line panitumumab studies. Ann. Oncol. 28, 1862-1868 (2017).

204. Petrelli, F. et al. Prognostic role of primary tumor location in non-metastatic gastric cancer: a systematic review and meta-analysis of 50 studies. Ann. Surg. Oncol. 24, 2655-2668 (2017).

205. Felismino, T. C. et al. Primary tumor location is a predictor of poor prognosis in patients with locally advanced esophagogastric cancer treated with perioperative chemotherapy. J. Gastrointest. Cance 51, 484-490 (2020)

206. Pêron, J. et al. The location of the primary colon cancer has no impact on outcomes in patients undergoing cytoreductive surgery for peritoneal metastasis. Surgery 165, 476-484 (2019).

207. Baratti, D. et al. Prognostic impact of primary side and RAS/RAF mutations in a surgical series of colorectal cancer with peritoneal metastases. Ann. Surg. Oncol. 28, 3332-3342 (2021).

208. Chang, L., Chang, M., Chang, H. M. \& Chang, F. Microsatellite instability: a predictive biomarker for cancer immunotherapy. Appl. Immunohistochem. Mol. Morphol. 26, E15-E21 (2018).

209. Dienstmann, R. \& Tabernero, J. Spectrum of gene mutations in colorectal cancer: implications for treatment. Cancer J. 22, 149-155 (2016).

210. Grieb, B. C. \& Agarwal, R. HER2-directed therapy in advanced gastric and gastroesophageal adenocarcinoma: triumphs and troubles. Curr. Treat. Opt. Oncol. 22, 88 (2021)

211. Greally, M., Kelly, C. M. \& Cercek, A. HER2 an emerging target in colorectal cancer. Curr. Probl. Cancer 42, 560-571 (2018)

212. Robson, M. E. et al. American society of clinical oncology policy statement update: genetic and genomic testing for cancer susceptibility. J. Clin Oncol. 33, 3660-3667 (2015).

213. Lheureux, S., Braunstein, M. \& Oza, A. M. Epithelia ovarian cancer: evolution of management in the era of precision medicine. CA Cancer J. Clin. 69, 280-304 (2019).

214. Guinney, J. et al. The consensus molecular subtypes of colorectal cancer. Nat. Med. 21, 1350-1356 (2015).

215. Sohn, B. H. et al. Clinical significance of four molecular subtypes of gastric cancer identified by the cancer genome atlas project. Clin. Cancer Res. 23, 4441-4449 (2017).
216. Ubink, I. et al. Histopathological and molecular classification of colorectal cancer and corresponding peritoneal metastases. Br. J. Surg. 105, e204-e211 (2018).

217. Körfer, J., Lordick, F. \& Hacker, U. T. Molecular targets for gastric cancer treatment and future perspectives from a clinical and translational point of view. Cancers 13, 5216 (2021)

218. Rubbia-Brandt, L. et al. Importance of histological tumor response assessment in predicting the outcome in patients with colorectal liver metastases treated with neo-adjuvant chemotherapy followed by liver surgery. Ann. Oncol. 18, 299-304 (2007).

219. Solass, W., Sempoux, C., Detlefsen, S., Carr, N. J. \& Bibeau, F. Peritoneal sampling and histological assessment of therapeutic response in peritoneal metastasis: proposal of the Peritoneal Regression Grading Score (PRGS). Pleura Peritoneum. 1, 99-107 (2016).

220. Solass, W. et al. Reproducibility of the peritoneal regression grading score for assessment of response to therapy in peritoneal metastasis. Histopathology 74, 1014-1024 (2019).

221. Böhm, S. et al. Chemotherapy response score: development and validation of a system to quantify histopathologic response to neoadjuvant chemotherapy in tubo-ovarian high-grade serous carcinoma. J. Clin. Oncol. 33, 2457-2463 (2015).

222. Böhm, S. et al. Histopathologic response to neoadjuvant chemotherapy as a prognostic biomarker in tubo-ovarian high-grade serous carcinoma: updated Chemotherapy Response Score (CRS) results. Int. J. Gynecol. Cancer 29, 353-356 (2019).

223. Pinto, D., Chandra, A., Crothers, B. A., Kurtycz, D. F. I. $\&$ Schmitt, F. The international system for reporting serous fluid cytopathology-diagnostic categories and clinical management. J. Am. Soc. Cytopathol. 9, 469-477 (2020)

224. Virgilio, E. et al. Gastric cancer cells in peritoneal lavage fluid: a systematic review comparing cytological with molecular detection for diagnosis of peritoneal metastases and prediction of peritoneal recurrences. Anticancer. Res. 38, 1255 (2018).

225. Honore, C., Goere, D., Souadka, A., Dumont, F. \& Elias, D. Definition of patients presenting a high risk of developing peritoneal carcinomatosis after curative surgery for colorectal cancer: a systematic review. Ann. Surg. Oncol. 20, 183-192 (2013).

226. Segelman, J., Akre, O., Gustafsson, U. O., Bottai, M. $\&$ Martling, A. Individualized prediction of risk of metachronous peritoneal carcinomatosis from colorectal cancer. Colorectal Dis. 16, 359-367 (2014).

227. Elias, D. et al. Results of systematic second-look surgery plus HIPEC in asymptomatic patients presenting a high risk of developing colorectal peritoneal carcinomatosis. Ann. Surg. 254, 289-293 (2011).

228. Tanaka, M. et al. Meta-analysis of recurrence pattern after resection for pancreatic cancer. Br. J. Surg. 106 1590-1601 (2019).

229. Yepuri, N., Bahary, N., Jain, A. \& Dhir, M. Review and update on the role of peritoneal cytology in the treatment of gastric cancer. J. Surg. Res. 235 607-614 (2019)

230. Yang, H. K. et al. Extensive peritoneal lavage with saline after curative gastrectomy for gastric cancer (EXPEL): a multicentre randomised controlled trial. Lancet Gastroenterol. Hepatol. 6, 120-127 (2021).

231. Klaver, C. E. L. et al. Adjuvant hyperthermic intraperitoneal chemotherapy in patients with locally advanced colon cancer (COLOPEC): a multicentre, open-label, randomised trial. Lancet Gastroenterol. Hepatol. 4, 761-770 (2019).

232. Goere, D. et al. Second-look surgery plus hyperthermic intraperitoneal chemotherapy versus surveillance in patients at high risk of developing colorectal peritoneal metastases (PROPHYLOCHIP-PRODIGE 15): a randomised, phase 3 study. Lancet Oncol. 21 , 1147-1154 (2020).

233. Ceelen, W. HIPEC with oxaliplatin for colorectal peritoneal metastasis: the end of the road? Eur. $J$. Surg. Oncol. 45, 400-402 (2019).

234. Arjona-Sanchez, A. et al. HIPECT4: multicentre randomized clinical trial to evaluate safety and efficacy of Hyperthermic intra-peritoneal chemotherapy (HIPEC) with Mitomycin C used during surgery for treatment of locally advanced colorectal carcinoma. BMC Cancer 18, 183 (2018).

235. Koga, S. et al. Prophylactic therapy for peritoneal recurrence of gastric cancer by continuous hyperthermic peritoneal perfusion with mitomycin C. Cancer 61, 232-237 (1988). 
236. Kaibara, N., Hamazoe, R., litsuka, Y., Maeta, M. \& Koga, S. Hyperthermic peritoneal perfusion combined with anticancer chemotherapy as prophylactic treatment of peritoneal recurrence of gastric cancer Hepatogastroenterology 36, 75-78 (1989).

237. Yonemura, Y. et al. Intraoperative chemohyperthermic peritoneal perfusion as an adjuvant to gastric cancer: final results of a randomized controlled study. Hepatogastroenterology 48, 1776-1782 (2001).

238. Brenkman, H. J. F., Paeva, M., van Hillegersberg, R. Ruurda, J. P. \& Haj Mohammad, N. Prophylactic hyperthermic intraperitoneal chemotherapy (HIPEC) for gastric cancer - a systematic review. J. Clin. Med. 8, 1685 (2019)

239. Cuzick, J. Preventive therapy for cancer. Lancet Oncol. 18, e472-e482 (2017)

240. Kastrinos, F., Samadder, N. J. \& Burt, R. W. Use of family history and genetic testing to determine risk of colorectal cancer. Gastroenterology 158, 389-403 (2020).

241. Hinchcliff, E. M., Bednar, E. M., Lu, K. H. \& Rauh-Hain, J. A. Disparities in gynecologic cancer genetics evaluation. Gynecol. Oncol. 153, 184-191 (2019).

242. Zauber, A. G. The impact of screening on colorectal cancer mortality and incidence: has it really made a difference? Dig. Dis. Sci. 60, 681-691 (2015).

243. Kim, J.-H. et al. Association between the National Cancer Screening Programme (NSCP) for gastric cancer and oesophageal cancer mortality. Br. J. Cancer 123, 480-486 (2020).

244. Kauff, N. D. et al. Risk-reducing salpingooophorectomy for the prevention of BRCA1- and BRCA2-associated breast and gynecologic cancer: a multicenter, prospective study. J. Clin. Oncol. 26, 1331-1337 (2008).

245. Rebbeck, T. R. et al. Prophylactic oophorectomy in carriers of BRCA1 or BRCA2 mutations. N. Engl. J. Med. 346, 1616-1622 (2002)

246. Guillem, J. G. et al. ASCO/SSO review of current role of risk-reducing surgery in common hereditary cancer syndromes. J. Clin. Oncol. 24, 4642-4660 (2006).

247. Seevaratnam, R. et al. A systematic review of the indications for genetic testing and prophylactic gastrectomy among patients with hereditary diffuse gastric cancer. Gastric Cancer 15 (Suppl. 1), S153-S163 (2012).

248. El Rami, F. E., Barsoumian, H. B. \& Khneizer, G. W. Hereditary diffuse gastric cancer therapeutic roadmap: current and novel approaches in a nutshell. Ther. Adv. Med. Oncol. 12, 1758835920967238 (2020).

249. Pandalai, P. K., Lauwers, G. Y., Chung, D. C., Patel, D. \& Yoon, S. S. Prophylactic total gastrectomy for individuals with germline CDH1 mutation. Surgery 149, 347-355 (2011).

250. Bleicher, J. \& Lambert, L. A. A palliative approach to management of peritoneal carcinomatosis and malignant ascites. Surg. Oncol. Clin. North. Am. 30 475-490 (2021)

251. Franko, J. et al. Treatment of colorectal peritoneal carcinomatosis with systemic chemotherapy: a pooled analysis of north central cancer treatment group phase III trials N9741 and N9841. J. Clin. Oncol. 30, 263-267 (2012)

252. Solass, W. et al. Morphology of the peritoneal cavity and pathophysiological consequences. Pleura Peritoneum. 1, 193-201 (2016)

253. Masoumi Moghaddam, S., Amini, A., Morris, D. L. \& Pourgholami, M. H. Significance of vascular endothelial growth factor in growth and peritoneal dissemination of ovarian cancer. Cancer Metastasis Rev. 31, 143-162 (2012)

254. Im, S. A. et al. Overall survival with ribociclib plus endocrine therapy in breast cancer. N. Engl. J. Med. 381, 307-316 (2019)

255. Bang, Y. J. et al. Trastuzumab in combination with chemotherapy versus chemotherapy alone for treatment of HER2-positive advanced gastric or gastro-oesophageal junction cancer (ToGA): a phase 3 open-label, randomised controlled trial. Lancet 376 , 687-697 (2010)

256. Swain, S. M. et al. Pertuzumab, trastuzumab, and docetaxel in HER2-positive metastatic breast cancer. N. Engl. J. Med. 372, 724-734 (2015).

257. Golan, T. et al. Maintenance olaparib for germline BRCA-mutated metastatic pancreatic cancer. N. Engl. J. Med. 381, 317-327 (2019).

258. Moore, K. et al. Maintenance olaparib in patients with newly diagnosed advanced ovarian cancer. N. Engl. J. Med. 379, 2495-2505 (2018).
259. Drilon, A. et al. Efficacy of larotrectinib in TRK fusion-positive cancers in adults and children. N. Engl. J. Med. 378, 731-739 (2018).

260. Andrê, T. et al. Pembrolizumab in microsatellite instability-high advanced colorectal cancer. N. Engl. J. Med. 383, 2207-2218 (2020)

261. Lemery, S., Keegan, P. \& Pazdur, R. First FDA approva agnostic of cancer site - when a biomarker defines the indication. N. Engl. J. Med. 377, 1409-1412 (2017).

262. Lordick, F. \& Janjigian, Y. Y. Clinical impact of tumour biology in the management of gastroesophageal cancer. Nat. Rev. Clin. Oncol. 13, 348-360 (2016).

263. Mateo, J. et al. A framework to rank genomic alterations as targets for cancer precision medicine: the ESMO Scale for Clinical Actionability of molecular Targets (ESCAT). Ann. Oncol. 29, 1895-1902 (2018).

264. Sugarbaker, P. H. Peritonectomy procedures. Cancer Treat. Res. 134, 247-264 (2007).

265. Dindo, D., Demartines, N. \& Clavien, P. A. Classification of surgical complications: a new proposal with evaluation in a cohort of 6336 patients and results of a survey. Ann. Surg. 240, 205-213 (2004).

266. National Cancer Institute. Cancer Therapy Evaluation Program. Common Terminology Criteria for Adverse Events v4.0 (CTCAE). National Cancer Institute http://ctep.cancer.gov/protocolDevelopment/ electronic_applications/ctc.htm (2020).

267. Arjona-Sanchez, A. et al. Laparoscopic cytoreductive surgery and hyperthermic intraperitoneal chemotherapy for limited peritoneal metastasis. The PSOGI international collaborative registry. Eur. J. Surg. Oncol. 47, 1420-1426 (2020).

268. Ansari, N. et al. Cytoreductive surgery and hyperthermic intraperitoneal chemotherapy in 1000 patients with perforated appendiceal epithelial tumours. Eur. J. Surg. Oncol. 42, 1035-1041 (2016).

269. Passot, G. et al. What made hyperthermic intraperitoneal chemotherapy an effective curative treatment for peritoneal surface malignancy: a 25-year experience with 1,125 procedures. J. Surg. Oncol. 113, 796-803 (2016).

270. Glehen, O., Mohamed, F. \& Gilly, F. N. Peritoneal carcinomatosis from digestive tract cancer: new management by cytoreductive surgery and intraperitoneal chemohyperthermia. Lancet Oncol. 5 219-228 (2004)

271. Armstrong, D. K. et al. Intraperitoneal cisplatin and paclitaxel in ovarian cancer. N. Engl. J. Med. 354 34-43 (2006)

272. Soucisse, M. L., Liauw, W., Hicks, G. \& Morris, D. L. Early postoperative intraperitoneal chemotherapy for lower gastrointestinal neoplasms with peritoneal metastasis: a systematic review and critical analysis Pleura Peritoneum. 4, 20190007 (2019).

273. Canbay, E. et al. Outcome data of patients with peritoneal carcinomatosis from gastric origin treated by a strategy of bidirectional chemotherapy prior to cytoreductive surgery and hyperthermic intraperitoneal chemotherapy in a single specialized center in Japan. Ann. Surg. Oncol. 21, 1147-1152 (2014).

274. Sugarbaker, P. H. \& Chang, D. Long-term regional chemotherapy for patients with epithelial malignant peritoneal mesothelioma results in improved survival. Eur. J. Surg. Oncol. 43, 1228-1235 (2017).

275. Charrier, T. et al. Cytoreductive surgery combined with hyperthermic intraperitoneal chemotherapy with oxaliplatin increases the risk of postoperative hemorrhagic complications: analysis of predictive factors. Ann. Surg. Oncol. 23, 2315-2322 (2016).

276. Siebert, M. et al. Severe hypersensitivity reactions to platinum compounds post-pressurized intraperitoneal aerosol chemotherapy (PIPAC): first literature report. Cancer Chemother. Pharmacol. 83, 425-2322 (2018).

277. Tan, G. H. C., Shannon, N. B., Chia, C. S., Soo, K. C. \& Teo, M. C. C. Platinum agents and mitomycin C-specific complications in cytoreductive surgery (CRS) and hyperthermic intraperitoneal chemotherapy (HIPEC) Int. J. Hyperth. 34, 595-600 (2018).

278. Alyami, M. et al. Ninety-day post-operative morbidity and mortality using the National Cancer Institute's common terminology criteria for adverse events better describe post-operative outcome after cytoreductive surgery and hyperthermic intraperitoneal chemotherapy. Int. J. Hyperth. 34, 532-537 (2017).

279. Lurvink, R. J. et al. The Delphi and GRADE methodology used in the PSOGI 2018 consensus statement on pseudomyxoma peritonei and peritoneal mesothelioma. Eur. J. Surg. Oncol. 47, 4-10 (2019).
280. Sugarbaker, P. H. New standard of care for appendiceal epithelial neoplasms and pseudomyxoma peritonei syndrome? Lancet Oncol. 7, 69-76 (2006).

281. Govaerts, K. et al. Appendiceal tumours and pseudomyxoma peritonei: literature review with PSOGI/EURACAN clinical practice guidelines for diagnosis and treatment. Eur. J. Surg. Oncol. 47 11-35 (2021)

282. Kusamura, S. et al. Multicentre study of the learning curve and surgical performance of cytoreductive surgery with intraperitoneal chemotherapy for pseudomyxoma peritonei. Br. J. Surg. 101 1758-1765 (2014)

283. Kusamura, S. et al. The role of hyperthermic intraperitoneal chemotherapy in pseudomyxoma peritonei after cytoreductive surgery. JAMA Surg. 156, e206363 (2021)

284. Reddy, S. et al. Extending the indications of intestinal transplantation - modified multi visceral transplantation for end-stage pseudomyxoma peritoneii. Transplantation 101, S89 (2017).

285. Pillai, K Akhter, J., Chua, T C \& Morris, D L Anticancer property of bromelain with therapeutic potential in malignant peritoneal mesothelioma. Cancer Invest. 31, 241-250 (2013).

286. Pillai, K., Ehteda, A., Akhter, J., Chua, T. C. \& Morris, D. L. Anticancer effect of bromelain with and without cisplatin or 5-FU on malignant peritoneal mesothelioma cells. Anticancer Drugs 25, 150-160 (2014).

287. Valle, S. J. et al. A novel treatment of bromelain and acetylcysteine (BromAc) in patients with peritoneal mucinous tumours: a phase I first in man study. Eur. J. Surg. Oncol. 47, 115-122 (2021).

288. Sgarbura, O. et al. Complete pathologic response after two-stage cytoreductive surgery with HIPEC for bulky pseudomyxoma peritonei: proof of concept. Int. J. Hyperth. 37, 585-591 (2020).

289. Malgras, B. et al. Impact of combination chemotherapy in peritoneal mesothelioma hyperthermic intraperitoneal chemotherapy (HIPEC): the RENAPE study. Ann. Surg. Oncol. 25, 3271-3279 (2018).

290. Simon, G. R. et al. Pemetrexed plus gemcitabine as first-line chemotherapy for patients with peritoneal mesothelioma: final report of a phase II trial. J. Clin. Oncol. 26, 3567-3572 (2008).

291. Zalcman, G. et al. Bevacizumab for newly diagnosed pleural mesothelioma in the mesothelioma avastin cisplatin pemetrexed study (MAPS): a randomised, controlled, open-label, phase 3 trial. Lancet 387 1405-1414 (2016).

292. Baas, P. et al. First-line nivolumab plus ipilimumab in unresectable malignant pleural mesothelioma (CheckMate 743): a multicentre, randomised, open-label, phase 3 trial. Lancet 397, 375-386 (2021).

293. Greenbaum, A. \& Alexander, H. R. Peritoneal mesothelioma. Transl. Lung Cancer Res. 9 (Suppl. 1), S120-S132 (2020).

294. Yan, T. D. et al. Cytoreductive surgery and hyperthermic intraperitoneal chemotherapy for malignant peritoneal mesothelioma: multi-institutional experience. J. Clin. Oncol. 27, 6237-6242 (2009).

295. Kusamura, S., Torres Mesa, P. A., Cabras, A., Baratti, D. \& Deraco, M. The role of Ki-67 and pre-cytoreduction parameters in selecting diffuse malignant peritoneal mesothelioma (DMPM) patients for cytoreductive surgery (CRS) and hyperthermic intraperitoneal chemotherapy (HIPEC). Ann. Surg. Oncol. 23, 1468-1473 (2016).

296. Alexander, H. R. et al. Treatment factors associated with long-term survival after cytoreductive surgery and regional chemotherapy for patients with malignant peritoneal mesothelioma. Surgery 153, 779-786 (2013).

297. Le Roy, F. et al. Conversion to complete cytoreductive surgery and hyperthermic intraperitoneal chemotherapy for malignant peritoneal mesothelioma after bidirectional chemotherapy. Ann. Surg. Oncol. 24, 3640-3646 (2017).

298. Alyami, M. et al. Unresectable peritoneal metastasis treated by pressurized intraperitoneal aerosol chemotherapy (PIPAC) leading to cytoreductive surgery and hyperthermic intraperitoneal chemotherapy. Eur. J. Surg. Oncol. 47, 128-133 (2021).

299. Sgarbura, O. et al. MESOTIP: phase II multicenter randomized trial evaluating the association of PIPAC and systemic chemotherapy vs. systemic chemotherapy alone as 1 st-line treatment of malignant peritoneal mesothelioma. Pleura Peritoneum. 4, 20190010 (2019). 
300. Elias, D. et al. Complete cytoreductive surgery plus intraperitoneal chemohyperthermia with oxaliplatin for peritoneal carcinomatosis of colorectal origin. J. Clin. Oncol. 27, 681-685 (2009)

301. Franko, J. et al. Cytoreductive surgery and hyperthermic intraperitoneal chemoperfusion versus systemic chemotherapy alone for colorectal peritoneal carcinomatosis. Cancer 116, 3756-3762 (2010).

302. Esquivel, J. et al. The American Society of Peritoneal Surface Malignancies (ASPSM) multiinstitution evaluation of the Peritoneal Surface Disease Severity Score (PSDSS) in 1,013 patients with colorectal cancer with peritoneal carcinomatosis. Ann. Surg. Oncol. 21, 4195-4201 (2014)

303. Cashin, P. H. et al. Cytoreductive surgery and intraperitoneal chemotherapy versus systemic chemotherapy for colorectal peritoneal metastases: a randomised trial. Eur. J. Cancer 53, 155-162 (2016).

304. Goere, D. et al. Results of a randomized phase 3 study evaluating the potential benefit of a second-look surgery plus HIPEC in patients at high risk of developing colorectal peritoneal metastases (PROPHYLOCHIP- NTC01226394). J. Clin. Oncol. 36 (Suppl. 15), 3531 (2018).

305. Abboud, K. et al. Management of colorectal peritoneal metastases: expert opinion. J. Visc. Surg. 156 377-379 (2019)

306. Al-Batran, S. E. et al. Effect of neoadjuvan chemotherapy followed by surgical resection on survival in patients with limited metastatic gastric or gastroesophageal junction cancer: the AIO-FLOT3 Trial. JAMA Oncol. 3, 1237-1244 (2017).

307. Bonnot, P. E. et al. Cytoreductive surgery with or without hyperthermic intraperitoneal chemotherapy for gastric cancer with peritoneal metastases (CYTO-CHIP study): a propensity score analysis. J. Clin. Oncol. 37, 2028-2040 (2019).

308. Chia, C. S. et al. Patients with peritoneal carcinomatosis from gastric cancer treated with cytoreductive surgery and hyperthermic intraperitoneal chemotherapy: is cure a possibility? Ann. Surg. Oncol. 23, 1971-1979 (2016).

309. Brandl, A., Yonemura, Y., Glehen, O., Sugarbaker, P. $\&$ Rau, B. Long term survival in patients with peritoneal metastasised gastric cancer treated with cytoreductive surgery and HIPEC: a multi-institutional cohort from PSOGI. Eur. J. Surg. Oncol. 47, 172-180 (2021).

310. Badgwell, B. et al. Phase II trial of laparoscopic hyperthermic intraperitoneal chemoperfusion for peritoneal carcinomatosis or positive peritoneal cytology in patients with gastric adenocarcinoma Ann. Surg. Oncol. 24, 3338-3344 (2017).

311. Beeharry, M. K. et al. Study protocol of a multicenter phase III randomized controlled trial investigating the efficiency of the combination of neoadjuvant chemotherapy (NAC) and neoadjuvant laparoscopic intraperitoneal hyperthermic chemotherapy (NLHIPEC) followed by R0 gastrectomy with intraoperative HIPEC for advanced gastric cancer (AGC): dragon II trial. BMC Cancer 20, 224 (2020).

312. Koemans, W. J. et al. Cytoreductive surgery and hyperthermic intraperitoneal chemotherapy versus palliative systemic chemotherapy in stomach cancer patients with peritoneal dissemination, the study protocol of a multicentre randomised controlled trial (PERISCOPE II). BMC Cancer 19, 420 (2019).

313. Alyami, M. et al. Pressurized intraperitoneal aerosol chemotherapy (PIPAC) for unresectable peritoneal metastasis from gastric cancer. Eur. J. Surg. Oncol. 47, 123-127 (2021)

314. Coccolini, F. et al. Complete versus incomplete cytoreduction in peritoneal carcinosis from gastric cancer, with consideration to $\mathrm{PCl}$ cut-off. Systematic review and meta-analysis. Eur. J. Surg. Oncol. 41, 911-919 (2015)

315. Coccolini, F. et al. Effect of intraperitoneal chemotherapy and peritoneal lavage in positive peritoneal cytology in gastric cancer. Systematic review and meta-analysis. Eur. J. Surg. Oncol. 42, 1261-1267 (2016).

316. Yonemura, Y. et al. Effects of neoadjuvant laparoscopic hyperthermic intraperitoneal chemotherapy and neoadjuvant intraperitoneal/systemic chemotherapy on peritoneal metastases from gastric cancer. Ann. Surg. Oncol. 24, 478-485 (2017).

317. Bristow, R. E., Tomacruz, R. S., Armstrong, D. K. Trimble, E. L. \& Montz, F. J. Survival effect of maximal cytoreductive surgery for advanced ovarian carcinoma during the platinum era: a meta-analysis. J. Clin. Oncol. 20, 1248-1259 (2002).
318. Vergote, I., Chiva, L. \& du Bois, A. Hyperthermic intraperitoneal chemotherapy in ovarian cancer. N. Engl. J. Med. 378, 1362-1363 (2018)

319. Kehoe, S. et al. Primary chemotherapy versus primary surgery for newly diagnosed advanced ovarian cancer (CHORUS): an open-label, randomised, controlled, non-inferiority trial. Lancet 386, 249-257 (2015).

320. Colombo, N. et al. ESMO-ESGO consensus conference recommendations on ovarian cancer: pathology and molecular biology, early and advanced stages, borderline tumours and recurrent diseaset. Ann. Oncol. 30, 672-705 (2019).

321. Harter, P. et al. A randomized trial of lymphadenectomy in patients with advanced ovarian neoplasms. N. Engl. J. Med. 380, 822-832 (2019).

322. Huo, Y. R., Richards, A., Liauw, W. \& Morris, D. L. Hyperthermic intraperitoneal chemotherapy (HIPEC) and cytoreductive surgery (CRS) in ovarian cancer: a systematic review and meta-analysis. Eur. J. Surg. Oncol. 41, 1578-1589 (2015).

323. Harter, P. et al. Surgery for recurrent ovarian cancer role of peritoneal carcinomatosis: exploratory analysis of the DESKTOP I Trial about risk factors, surgical implications, and prognostic value of peritoneal carcinomatosis. Ann. Surg. Oncol. 16, 1324-1330 (2009).

324. Harter, P. et al. Surgery in recurrent ovarian cancer: the Arbeitsgemeinschaft Gynaekologische Onkologie (AGO) DESKTOP OVAR trial. Ann. Surg. Oncol. 13, 1702-1710 (2006)

325. Fanfani, F. et al. Is there a role for tertiary (TCR) and quaternary (OCR) cytoreduction in recurrent ovarian cancer? Anticancer. Res. 35, 6951-6955 (2015).

326. Shi, T. et al. Secondary cytoreduction followed by chemotherapy versus chemotherapy alone in platinum-sensitive relapsed ovarian cancer (SOC-1): a multicentre, open-label, randomised, phase 3 trial. Lancet Oncol. https://doi.org/10.1016/S14702045(21)00006-1 (2021)

327. Spiliotis, J. et al. The role of cytoreductive surgery and hyperthermic intraperitoneal chemotherapy in the management of recurrent advanced ovarian cancer: a prospective study. J. Buon. 16, 74-79 (2011).

328. Bakrin, N. et al. Peritoneal carcinomatosis treated with cytoreductive surgery and Hyperthermic Intraperitoneal Chemotherapy (HIPEC) for advanced ovarian carcinoma: a French multicentre retrospective cohort study of 566 patients. Eur. J. Surg. Oncol. 39, 1435-1443 (2013).

329. US National Library of Medicine. ClinicalTrials.gov https://clinicaltrials.gov/ct2/show/NCT03220932? term $=$ NCT03220932 \&draw $=2$ \&rank=1 (2019)

330. Tempfer, C. B. et al. A phase I, single-arm, open-label, dose escalation study of intraperitoneal cisplatin and doxorubicin in patients with recurrent ovarian cancer and peritoneal carcinomatosis. Gynecol. Oncol. 150, 23-30 (2018).

331. Bakrin, N. et al. PIPAC-OV3: a multicenter, open-label, randomized, two-arm phase III trial of the effect on progression-free survival of cisplatin and doxorubicin as pressurized intra-peritoneal aerosol chemotherapy (PIPAC) vs. chemotherapy alone in patients with platinum-resistant recurrent epithelial ovarian, fallopian tube or primary peritoneal cancer. Pleura Peritoneum. 3, 20180114 (2018).

332. Tempfer, C. B., Hilal, Z., Dogan, A., Petersen, M \& Rezniczek, G. A. Concentrations of cisplatin and doxorubicin in ascites and peritoneal tumor nodules before and after pressurized intraperitoneal aerosol chemotherapy (PIPAC) in patients with peritoneal metastasis. Eur. J. Surg. Oncol. 44, 1112-1117 (2018).

333. Goéré, D. et al. Complete cytoreductive surgery plus HIPEC for peritoneal metastases from unusual cancer sites of origin: results from a worldwide analysis issue of the Peritoneal Surface Oncology Group International (PSOGI). Int. J. Hyperth. 33, 520-527 (2017).

334. Amblard, I. et al. Cytoreductive surgery and HIPEC improve survival compared to palliative chemotherapy for biliary carcinoma with peritoneal metastasis: a multi-institutional cohort from PSOGI and BIG RENAPE groups. Eur. J. Surg. Oncol. 44, 1378-1383 (2018).

335. Mehta, S. et al. Is there an oncological interest in the combination of CRS/HIPEC for peritoneal carcinomatosis of HCC? Results of a multicenter international study. Eur. J. Surg. Oncol. 44 1786-1792 (2018).

336. Farma, J. M. et al. Limited survival in patients with carcinomatosis from foregut malignancies after cytoreduction and continuous hyperthermic peritoneal perfusion. J. Gastrointest. Surg. 9, 1346-1353 (2005).

337. Cardi, M. et al. Treatment of peritoneal carcinomatosis from breast cancer by maximal cytoreduction and HIPEC: a preliminary report on 5 cases. Breast 22 845-849 (2013)

338. McLemore, E. C. et al. Breast cancer: presentation and intervention in women with gastrointestinal metastasis and carcinomatosis. Ann. Surg. Oncol. 12, 886-894 (2005).

339. Rossi, C. R. et al. Hyperthermic intraperitoneal intraoperative chemotherapy after cytoreductive surgery for the treatment of abdominal sarcomatosis: clinical outcome and prognostic factors in 60 consecutive patients. Cancer 100, 1943-1950 (2004).

340. Baratti, D. et al. Peritoneal sarcomatosis: is there a subset of patients who may benefit from cytoreductive surgery and hyperthermic intraperitoneal chemotherapy? Ann. Surg. Oncol. 17, 3220-3228 (2010)

341. Salti, G. I. Ailabouni, L \& Undevia, S. Cytoreductive surgery and hyperthermic intraperitoneal chemotherapy for the treatment of peritoneal sarcomatosis. Ann. Surg. Oncol. 19, 1410-1415 (2012).

342. Baumgartner, J. M. et al. Aggressive locoregional management of recurrent peritoneal sarcomatosis. J. Surg. Oncol. 107, 329-334 (2013).

343. Sommariva, A. et al. Cytoreductive surgery and hyperthermic intraperitoneal chemotherapy in patients with peritoneal sarcomatosis: long-term outcome from a single institution experience. Anticancer. Res. 33, 3989-3994 (2013).

344. Olesiński, T. Cytoreductive surgery and HIPEC in the treatment of peritoneal metastases of sarcomas and other rare malignancies. Pol. Prz. Chirurgiczny. 89, 31-36 (2017)

345. Norlén, O. et al. Long-term results of surgery for small intestinal neuroendocrine tumors at a tertiary referral center. World J. Surg. 36, 1419-1431 (2012).

346. Norlén, O. et al. Peritoneal carcinomatosis from small intestinal neuroendocrine tumors: clinical course and genetic profiling. Surgery 156, 1512-1521 (2014).

347. Pavel, M. et al. ENETS consensus guidelines update for the management of distant metastatic disease of intestinal, pancreatic, bronchial neuroendocrine neoplasms (NEN) and NEN of unknown primary site. Neuroendocrinology 103, 172-185 (2016).

348. Elias, D. et al. Neuroendocrine carcinomas: optimal surgery of peritoneal metastases (and associated intra-abdominal metastases). Surgery $155,5-12$ (2014).

349. Mayrbaurl, B. et al. Quality of life across chemotherapy lines in patients with advanced colorectal cancer: a prospective single-center observational study. Support Care Cancer 24, 667-674 (2016).

350. Corn, B. W., Feldman, D. B. \& Wexler, I. The science of hope. Lancet Oncol. 21, e452-e459 (2020).

351. Kaasa, S. \& Loge, J. H. Quality-of-life assessment in palliative care. Lancet Oncol. 3, 175-182 (2002).

352. Lambert, L. A. \& Wiseman, J. Palliative management of peritoneal metastases. Ann. Surg. Oncol. 25, 2165-2171 (2018).

353. Odendahl, K. et al. Quality of life of patients with end-stage peritoneal metastasis treated with pressurized intraperitoneal aerosol chemotherapy (PIPAC). Eur. J. Surg. Oncol. 41, 1379-1385 (2015).

354. Franko, J. et al. Prognosis of patients with peritoneal metastatic colorectal cancer given systemic therapy: an analysis of individual patient data from prospective randomised trials from the Analysis and Research in Cancers of the Digestive System (ARCAD) database. Lancet Oncol. 17, 1709-1719 (2016).

355. Prigerson, H. G. et al. Chemotherapy use, performance status, and quality of life at the end of life. JAMA Oncol. 1, 778-784 (2015).

356. Enzinger, A. C., Zhang, B., Schrag, D. \& Prigerson, H. C. Outcomes of prognostic disclosure: associations with prognostic understanding, distress, and relationship with physician among patients with advanced cancer. J. Clin. Oncol. 33, 3809-3816 (2015).

357. Weeks, J. C. et al. Patients' expectations about effects of chemotherapy for advanced cancer. N. Engl. J. Med. 367, 1616-1625 (2012)

358. Francescutti, V. A. et al. Characterizing the patient experience of CS/HIPEC through in-depth interviews with patients: identification of key concepts in the development of a patient-centered program. Ann. Surg. Oncol. 26, 1063-1070 (2019). 
359. Tan, W. J. et al. Quality of life after cytoreductive surgery and hyperthermic intraperitonea chemotherapy: an Asian perspective. Ann. Surg. Oncol. 20, 4219-4223 (2013)

360. Roudijk, B., Donders, R. \& Stalmeier, P. Cultural values: can they explain self-reported health? Qual. Life Res. 26, 1531-1539 (2017)

361. Cashin, P. H. et al. Quality of life and cost effectiveness in a randomized trial of patients with colorectal cancer and peritoneal metastases. Eur. J. Surg. Oncol. 44, 983-990 (2018)

362. Stearns, A. T. et al. Long-term quality of life after cytoreductive surgery and heated intraperitoneal chemotherapy for pseudomyxoma peritonei: a prospective longitudinal study. Ann. Surg. Oncol. 25, 965-973 (2018)

363. Koole, S. N. et al. Health-related quality of life after interval cytoreductive surgery with or without hyperthermic intraperitoneal chemotherapy (HIPEC) in patients with stage III ovarian cancer. Eur. J. Surg. Oncol. 47, 101-107 (2021).

364. Dodson, R. M. et al. Quality-of-life evaluation after cytoreductive surgery with hyperthermic intraperitoneal chemotherapy. Ann. Surg. Oncol. 23 (Suppl. 5), 772-783 (2016)

365. Zhu, Y., Hanna, N., Boutros, C. \& Alexander, H. R. Jr. Assessment of clinical benefit and quality of life in patients undergoing cytoreduction and hyperthermic intraperitoneal chemotherapy (HIPEC) for management of peritoneal metastases. J. Gastrointest. Oncol. 4, 62-71 (2013).

366. Shan, L. Saxena, A., Shan, B. L. \& Morris, D. L. Quality of life after cytoreductive surgery and hyperthermic intra-peritoneal chemotherapy for peritoneal carcinomatosis: a systematic review and meta-analysis. Surg. Oncol. 23, 199-210 (2014).

367. Verduin, M., Hoeben, A., De Ruysscher, D. \& Vooijs, M Patient-derived cancer organoids as predictors of treatment response. Front. Oncol. 11, 820 (2021)

368. Schutgens, F. \& Clevers, H. Human organoids: tools for understanding biology and treating diseases. Annu. Rev. Pathol. 15, 211-234 (2020).

369. Fujii, M. \& Sato, T. Somatic cell-derived organoids as prototypes of human epithelial tissues and diseases. Nat. Mater. 20, 156-169 (2020).

370. Brodeur, M. N. et al. Carboplatin response in preclinical models for ovarian cancer: comparison of 2D monolayers, spheroids, ex vivo tumors and in vivo models. Sci. Rep. 11, 1-12 (2021).

371. Ceelen, W., Braet, H., Van Ramshorst, G., Willaert, W. \& Remaut, K. Intraperitoneal chemotherapy for peritoneal metastases: an expert opinion. Expert Opin. Drug Deliv. 17, 511-522 (2020).

372. Polack, F. P. et al. Safety and efficacy of the BNT162b2 mRNA Covid-19 vaccine. N. Engl. J. Med. 383, 2603-2615 (2020)

373. Baden, L. R. et al. Efficacy and safety of the mRNA-1273 SARS-CoV-2 vaccine. N. Engl. J. Med. 384, 403-416 (2021).

374. Sugarbaker, P. H. Intraperitoneal paclitaxel: pharmacology, clinical results and future prospects. J. Gastrointest. Oncol. 12 (Suppl. 1), S231 (2021).

375. Dakwar, G. R. et al. Nanomedicine-based intraperitoneal therapy for the treatment of peritoneal carcinomatosis - mission possible? Adv. Drug Deliv. Rev. 108, 13-24 (2017).

376. Levine E. \& Ceelen W. P. In Biomaterials and Drug Delivery Systems for Intraperitoneal Chemotherapy Ch. 32 (Taylor \& Francis Group, 2016)

377. Dakwar G. \& Remaut, K. In Intraperitoneal Nonviral Nucleic Acid Delivery in the Treatment of Peritoneal Cancer Ch. 32 (Taylor \& Francis Group, 2016).

378. Shariati, M., Willaert, W., Ceelen, W., De Smedt, S. C. \& Remaut, K. Aerosolization of nanotherapeutics as a newly emerging treatment regimen for peritoneal carcinomatosis. Cancers 11, 906 (2019).

379. Shariati, M. et al. Synergy between intraperitoneal aerosolization (PIPAC) and cancer nanomedicine: cisplatin-loaded polyarginine-hyaluronic acid nanocarriers efficiently eradicate peritoneal metastasis of advanced human ovarian cancer. ACS Appl. Mater Interfaces 12, 29024-29036 (2020).

380. Shariati, M. et al. High pressure nebulization (PIPAC) versus injection for the intraperitoneal administration of mRNA complexes. Pharm. Res. 36, 1-13 (2019).

381. Chen, C.-H. et al. Thermosensitive injectable hydrogel for simultaneous intraperitoneal delivery of doxorubicin and prevention of peritoneal adhesion. Int. J. Mol. Sci. 19, 1373 (2018).

382. Song, L. et al. Peritoneal adhesion prevention with a biodegradable and injectable N,O-carboxymethyl chitosan-aldehyde hyaluronic acid hydrogel in a rat repeated-injury model. Sci. Rep. 6, 1-13 (2016).

383. Bhatt A. In Management of Peritoneal Metastases Cytoreductive Surgery, HIPEC and Beyond 469-506 (Springer, 2018).

384. Van Oudheusden, T. R. Targeting the peritoneum with novel drug delivery systems - review peritoneal carcinomatosis. Anticancer Res. 35, 627-634 (2015).

385. Gao, X. et al. Novel thermosensitive hydrogel for preventing formation of abdominal adhesions. Int. J. Nanomed. 8, 2453-2463 (2013).

386. De Smet, L., Ceelen, W., Remon, J. P. \& Vervaet, C. Optimization of drug delivery systems for intraperitoneal therapy to extend the residence time of the chemotherapeutic agent. ScientificWorldJournal 2013, 720858 (2013)

387. Padmakumar, S., Parayath, N. N., Nair, S. V., Menon, D. \& Amiji, M. M. Enhanced anti-tumor efficacy and safety with metronomic intraperitoneal chemotherapy for metastatic ovarian cancer using biodegradable nanotextile implants. J. Controlled Rel. 305, 29-40 (2019).

388. Bommareddy, P. K., Shettigar, M. \& Kaufman, H. L. Integrating oncolytic viruses in combination cancer immunotherapy. Nat. Rev. Immunol. 18, 498-513 (2018).

389. Liu, Z., Ravindranathan, R., Kalinski, P., Guo, Z. S. $\&$ Bartlett, D. L. Rational combination of oncolytic vaccinia virus and PD-L1 blockade works synergistically to enhance therapeutic efficacy. Nat. Commun. 8, 14754 (2017)

390. Cohn, D. E. et al. Randomized phase IIB evaluation of weekly paclitaxel versus weekly paclitaxel with oncolytic reovirus (Reolysin ${ }^{\circledR}$ ) in recurrent ovarian, tubal, or peritoneal cancer: an NRG Oncology/ Gynecologic Oncology Group study. Gynecol. Oncol. 146, 477-483 (2017)

391. Giehl, E. et al. In vivo priming of peritoneal tumor reactive lymphocytes with a potent oncolytic virus for adoptive cell therapy. Front. Immunol. 12, 610042 (2021).

392. Lee, Y. S. et al. Oncolytic vaccinia virus reinvigorates peritoneal immunity and cooperates with immune checkpoint inhibitor to suppress peritoneal carcinomatosis in colon cancer. J. Immunother. Cancer 8, e000857 (2020)

393. Bhatt, A. \& Glehen, O. Extent of peritoneal resection for peritoneal metastases: looking beyond a complete cytoreduction. Ann. Surg. Oncol. 27, 1458-1470 (2020).

394. Dip, $\mathrm{F}$ et al Consensus conference statement on the general use of near-infrared fluorescence imaging and indocyanine green guided surgery: results of a modified Delphi study. Ann. Surg. https://doi.org/ 10.1097/SLA.0000000000004412 (2020).

395. Liberale, $\mathrm{G}$ et al. Fluorescence imaging after indocyanine green injection for detection of peritoneal metastases in patients undergoing cytoreductive surgery for peritoneal carcinomatosis from colorectal cancer. Ann. Surg. 264, 1110-1115 (2016).

396. Jiao, J. et al. Quicker, deeper and stronger imaging. a review of tumor-targeted, near-infrared fluorescent dyes for fluorescence guided surgery in the preclinical and clinical stages. Eur. J. Pharmaceutics Biopharmaceutics. 152, 123-143 (2020).

397. Van Dam, G. M. et al. Intraoperative tumor-specific fluorescence imaging in ovarian cancer by folate receptor-a targeting: first in-human results. Nat. Med. 17, 1315-1319 (2011)

398. Farghaly, S. A. Single Port Access (SPA) Robot assisted laparoscopic partial parietal peritonectomy in patients with advanced and recurrent ovarian cancer: Farghaly's technique. J. Minim. Invasive Gynecol. 19, S157 (2012)

399. Chen, T. C. \& Liang, J. T. Robotic low anterior resection with total hysterectomy, bilateral salpingooophorectomy and en bloc pelvic peritonectomy followed by hyperthermic intraperitoneal chemotherapy for the multi-modal treatment of rectosigmoid cancer with peritoneal seeding - a video vignette. Colorectal Dis. 23, 1591-1592 (2021).

400. Reymond, M. Demtroeder, C. Solass, W Winnekendonk, G. \& Tempfer, C. Electrostatic precipitation pressurized intraperitoneal aerosol chemotherapy (ePIPAC): first in-human application. Pleura Peritoneum 1, 109-116 (2016).

401. Kakchekeeva, T. et al. In vivo feasibility of electrostatic precipitation as an adjunct to pressurized intraperitoneal aerosol chemotherapy (ePIPAC) Ann. Surg. Oncol. 23, 592-598 (2016).
402. Bachmann, C. et al. Technology development of hyperthermic pressurized intraperitoneal aerosol chemotherapy (hPIPAC). Surgical Endosc. 35, 6358-6365 (2021).

403. Bushati, M. et al. The current practice of cytoreductive surgery and HIPEC for colorectal peritoneal metastases: Results of a worldwide web-based survey of the Peritoneal Surface Oncology Group International (PSOGI). Eur. J. Surg. Oncol. 44, 1942-1948 (2018).

404. Yurttas, C et al. Systematic review of variations in hyperthermic intraperitoneal chemotherapy (HIPEC) for peritoneal metastasis from colorectal cancer. J. Clin. Med. 7, 567 (2018).

405. Cremolini, C. et al. Surrogate endpoints in second-line trials of targeted agents in metastatic colorectal cancer: a literature-based systematic review and meta-analysis. Cancer Res. Treat. 49, 834-845 (2017).

406. Kipps, E., Tan, D. \& Kaye, S. Meeting the challenge of ascites in ovarian cancer: new avenues for therapy and research. Nat. Rev. Cancer 13, 273-282 (2013).

\section{Acknowledgements}

The authors thank Jeanine for their contribution in Box 2 .

\section{Author contributions}

Introduction (D.C.-G., M.H. and F.L.); Epidemiology (D.C.-G. M.H., A.B., K.K.T. and M.C.); Mechanisms/Pathophysiology (D.C.-G., M.H., W.C. and R.R.); Diagnosis/Screening/ Prevention (D.C.-G., M.H., M.A., O.G., F.L., O.S. and K.V.D.S.); Management (D.C.-G., M.H., M.A., O.G. and F.L.); Quality of life (D.C.-G., M.H., A.B., O.S. and K.V.D.S.); Outlook (D.C.-G., M.H., W.C. and R.R.); Overview of the Primer (D.C.-G.).

\section{Competing interests}

R.R. has received group research and engineering support from Fisher and Paykel. K.V.D.S. is a board member of the European Society of Surgical Oncology (ESSO) and an executive committee member of the Peritoneal Surface Oncology Group International (PSOGI). D.C.-G. holds honorary functions within ESSO as member of the Boards of directors, the Spanish Society of Surgical Oncology (SEOQ) as member of the Boards of Directors, the Spanish Federation of Oncologic Societies (FESEO) as secretary and member of the Boards of Directors, the International Society for the Study of Pleura and Peritoneum (ISSPP) as member of the communication committee, and the Enhanced Recovery After Surgery (ERAS) society and is responsible for the ERAS HIPEC website content. A.B. is on the review committee of the Department of Science and Technology of India, and declares no financial or non-financial competing interests. M.A. is a member of the ISSPP Education committee. M.H. has received research funding (institution) from Nestlé health science, speaker honorary (institution) from MSD, Nestlé, Fresenius, Capnomed and Encare, is a board member of the ERAS society and is chair of education of the ISSPP O.G is a consultant for GAMIDA (financial competing interest), Director of RENAPE, and President of BIG RENAPE association (non-financial competing interests). O.S. holds honorary functions with ESSO (member of the Communication committee), ISSPP (Public Relations committee), the French Society of Surgical Oncology (executive board), the French National Network for Peritoneum (RENAPE; board member) and the Romanian Students' Society of Surgery (honorary president). W.C. is Board member of the Belgian Society of Surgical Oncology and Royal Belgian Society of Surgery, and Editor-in-Chief of Acta Chirurgica Belgica, and Associate editor of European Surgical Research and International Journal of Hyperthermia. F.L., K.K.T. and M.C. declares no competing interests.

\section{Peer review information}

Nature Reviews Disease Primers thanks Peter Cashin, Yasuhiro Kodera, Winston Spencer Liauw, Beate Rau and the other, anonymous, reviewer(s) for their contribution to the peer review of this work.

Publisher's note

Springer Nature remains neutral with regard to jurisdictional claims in published maps and institutional affiliations.

Supplementary information

The online version contains supplementary material available at https://doi.org/10.1038/s41572-021-00326-6.

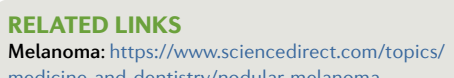

medicine-and-dentistry/nodular-melanom

C) Springer Nature Limited 2021 